\title{
Belonging-Centered Instruction: An Observational Approach toward Establishing Inclusive Mathematics Classrooms
}

\author{
Jamaal Sharif Matthews \\ DeLeon L. Gray \\ Qiana Lachaud \\ Tamika L. McElveen \\ Xiao-Yin Chen \\ Tiana Victor \\ Eric Okai \\ Kyle Boomhower \\ Jingyun Wu \\ Eunkyoung Cha
}




\section{Table of Contents:}

Belonging-Centered Instruction: An Observational Approach toward Establishing Inclusive Mathematics Classrooms.

Introduction: 5

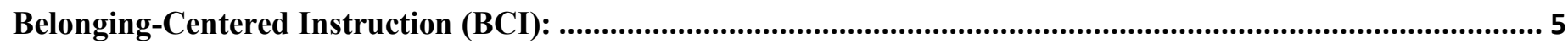

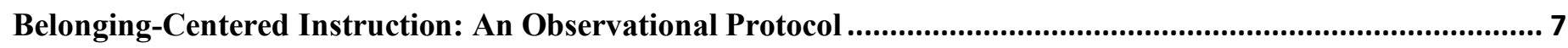

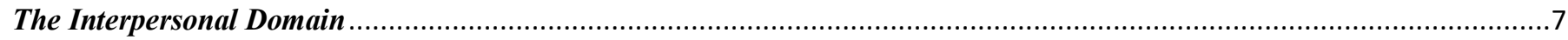

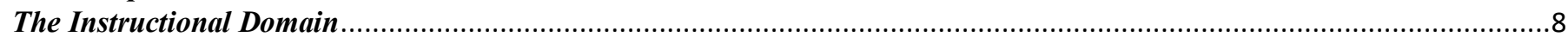

Development of the Belonging-Centered Instruction Observational Protocol...................................................... 9

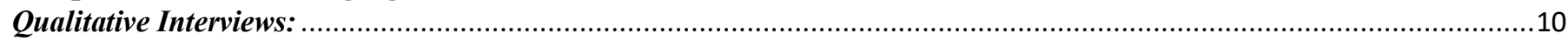

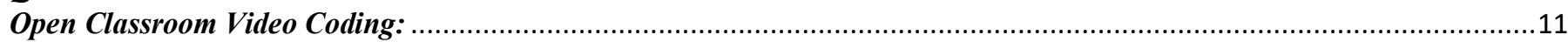

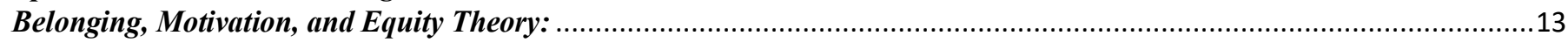

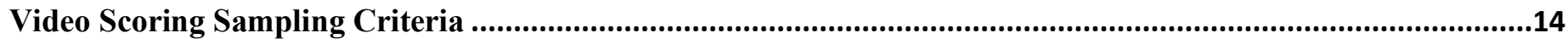

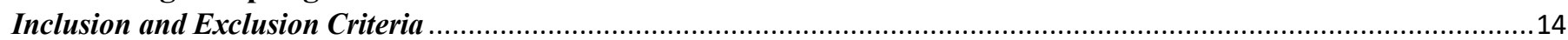

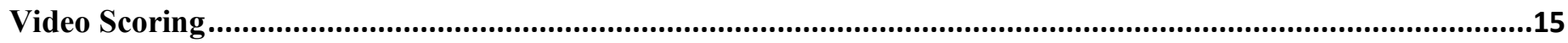

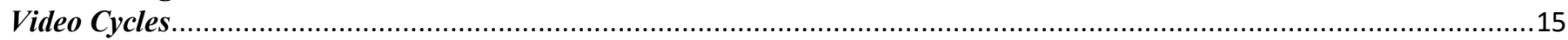

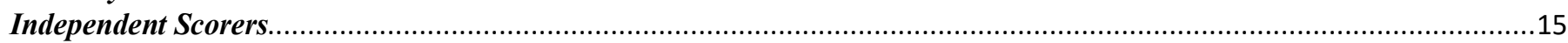

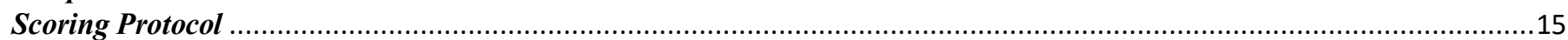

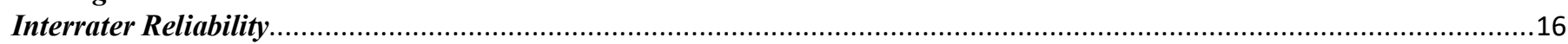

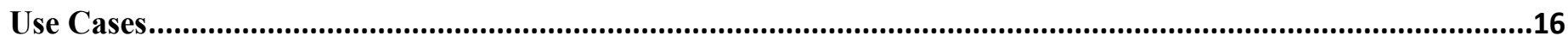

Use Case 01: Using the BCI Protocol to Predict Student Outcomes ..............................................................17

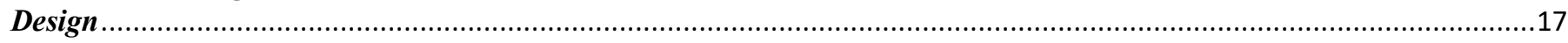

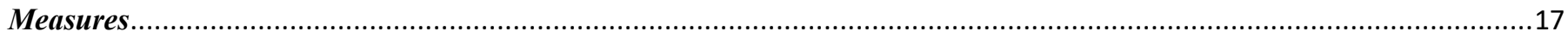

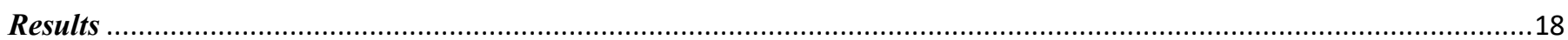

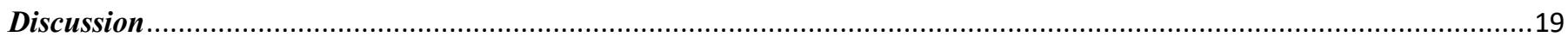

Use Case 02: Using the BCI Protocol to Document the Dynamic Interplay of Interpersonal and Instructional

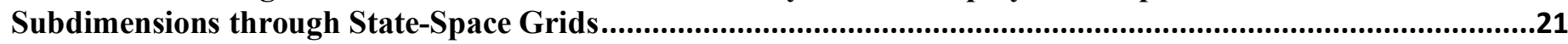

Method

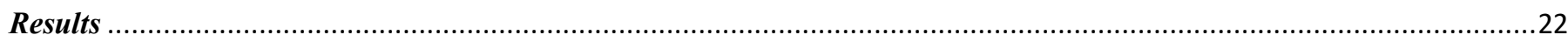

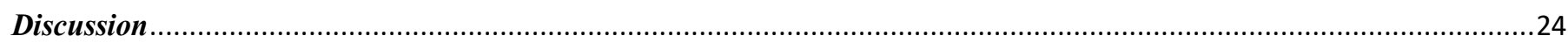

Belonging-Centered Instruction (BCI) Observational Protocol.....................................................25

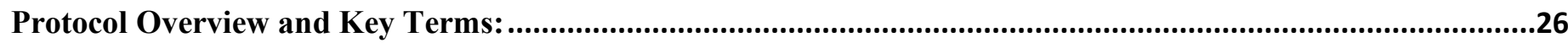

Procedures for Observing and Scoring Classrooms Using the BCI Observational Protocol: ...............................28

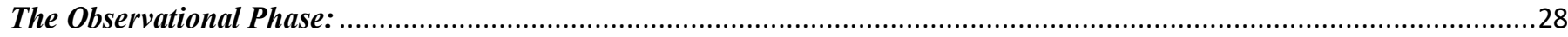

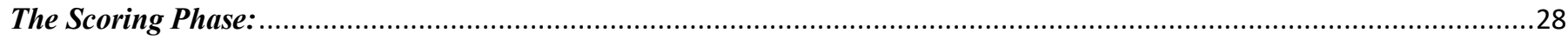

Quick Start Guide to Observational Scoring......................................................................................36

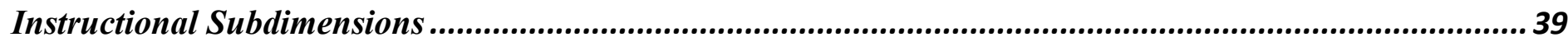

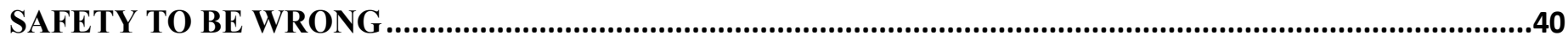

Scoring Details:

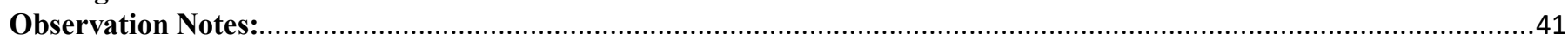

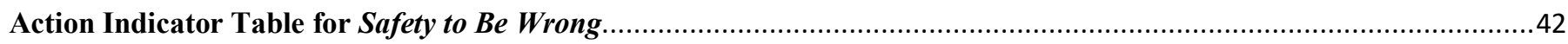

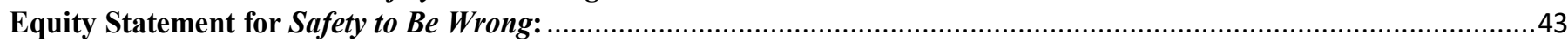

**Scoring Sheets for all Instructional and Interpersonal subdimensions is available in full version only**

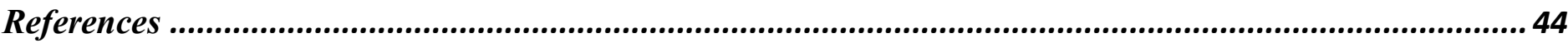


Belonging-Centered Instruction: An Observational Approach toward Establishing Inclusive Mathematics Classrooms 


\section{Introduction:}

Historically, students of color have faced persistent threats to their sense of belonging in U.S. schools and classrooms. As a consequence of disproportionately attending schools that 1) are segregated and under-resourced (DarlingHammond, 2013; Frankenberg, 2013), 2) maintain harsh suspension and expulsion policies (Scott et al., 2017), and 3) offer few honors and advanced placement courses (Ferguson et al., 2001; Klugman, 2013; Malkus, 2016; Reyes, 2006; Solórzano \& Ornelas, 2004), Black and Latinx students - compared to their White peers - must often navigate punitive and inequitable school environments that undermine their sense of belongingness. Further, Black and Latinx students regularly encounter curricula and textbooks that marginalize and fail to reflect their culture (Brown \& Brown, 2010; Sleeter \& Grant, 1991); the repression of their cultural expressions such as language, fashion, and hairstyles (Blau, 2003; Emdin, 2010; Kirkland \& Jackson, 2009; Mitchell, 2013); and stereotypes about their academic ability and intelligence that in turn reinforce low teacher expectations and outright discrimination (Nasir \& Shah, 2011; Okeke et al., 2009; Pena-Shaff et al., 2019). These various forms of cultural stigmatization can have a cumulative effect resulting in Black and Latinx students questioning whether they belong in their schools and classrooms.

Beyond these typical obstacles to belonging for students of color in schools, additional threats can arise in middle school mathematics classrooms. Research has shown that Black and Latinx students' sense of belonging decreases throughout the middle school years (Cook et al., 2012; Hughes et al., 2015; Wang \& Eccles, 2012), especially in mathematics (Hamm \& Faircloth, 2005). Among the various contributors to this decline, middle school Black and Latinx students must navigate the increasing rigor and abstract density of mathematics (Hidi, 2000) while also coming to consciousness of their racial identity and the stigma associated with it (i.e., what they and others think about their race, and how those perceptions shape their past, present, and future) (Umaña-Taylor et al., 2014). In other words, Black and Latinx students have to make meaning of mathematics and its ever-growing complexity and abstractness while negotiating stigma, stress, and discrimination in segregated, punitive, and impersonal school settings (Eccles \& Roeser, 2011; Huang \& Stormshak, 2011).

In addition, the systemic conception of mathematics as "culture-free," "colorblind," or distinct from cultural values (Battey, 2013; Martin et al., 2010) ultimately masks and preserves the white supremacist status quo that informs how mathematics is taught and evaluated (Battey, 2013; Martin, 2013, 2019), as well as who can and cannot do mathematics (Stinson, 2004, 2013). For example, many programs and curricula designed to promote equity in math education (e.g., Mathematics for All, Common Core) still prioritize white values and contributions while neglecting those of Black and Latinx individuals and communities (Martin, 2008). They also encourage Black and Latinx students to assimilate to white standards of achievement and white constructions of mathematical language (Martin, 2013, 2015). These factors can make Black and Latinx students reticent to engage more deeply in mathematics (Pauker et al., 2015), and can also undermine their sense of belonging in math classrooms, spaces, and professions.

Altogether, the disenfranchisement that Black and Latinx students face in mathematics classrooms illustrates a pernicious assault on their sense of belonging as mathematical thinkers and doers. Since belonging is a fundamental human need (Baumeister \& Leary, 1995; Maslow, 1943) linked with a variety of adaptive academic effects such as intrinsic motivation, self-efficacy, persistence, and achievement (Adelabu, 2007; Boston \& Warren, 2017; Freeman et al., 2007; Goodenow \& Grady, 1993; Sánchez et al., 2005; Strayhorn, 2020; Walton \& Cohen, 2007, 2011), these threats to Black and Latinx students' sense of belongingness in schools (and especially in middle school mathematics classrooms) contribute to a host of inequitable academic experiences and outcomes for them (Darling-Hammond, 2013; Gutiérrez, 2012; National Center for Education Statistics [NCES], 2020). In order to promote learning equity for Black and Latinx students in middle school mathematics classrooms, there is a significant need to center their sense of belonging in future research and praxis.

\section{Belonging-Centered Instruction (BCI):}

To address the alienation many students, particularly students of color, experience in mathematics classrooms, the research team conceptualizes a pedagogical framework for establishing inclusive mathematical learning environments: Belonging-Centered Instruction (BCI). In this work, Belonging-Centered Instruction is defined as teachers' provision of opportunities for active inclusion, achievement, identification, and empowerment via interpersonal interactions and instructional techniques (Goodenow, 1993; Gutiérrez, 2012). While belonging is a multidimensional construct shaped by various factors including peer interactions (Booker, 2007; Delgado et al., 2016; Juvonen, 2006), institutional systems (Bottiani et al., 2017; Kogachi \& Graham, 2020), and family involvement (Mo \& Singh, 2008; Wang \& Eccles, 2012), research has indicated that teacher support is one of the strongest predictors of students' sense of belonging (Allen et al., 2018). Because of the power and access teachers possess in meeting students' belongingness needs, Belonging-Centered Instruction is emphasized as the essential provision of social and pedagogical opportunities that mitigate students' feelings of alienation, exclusion, and dehumanization (i.e., experiencing the racial and cultural oppression in mathematical spaces that they encounter in broader society; Bartolomé, 1994; Gutiérrez, 2018) in mathematics classrooms. 
In this project, Belonging-Centered Instruction is conceptualized as two domains of teacher actions that encourage student belonging: interpersonal supports and instructional supports. Interpersonal supports for belonging refer to teacher practices that actively facilitate social ties, social comfort, and interconnectedness between members of a classroom. Research indicates the importance of interpersonal teacher qualities in shaping student belonging (e.g., Anderman, 2003; Furrer \& Skinner, 2003; Martin \& Dowson, 2009; Wentzel, 1998, 1999), especially among students from historically marginalized groups (e.g., Booker, 2004; Garcia-Reid, 2007; Maloney \& Matthews, 2020). Some research has specifically identified teacher warmth, emotional support, and emphasis on caring relationships as strong predictors of students' classroom belonging (Allen et al., 2018; Hughes, 2011). Maloney and Matthews (2020) found that students felt more connected within the classroom when their teachers authentically sought to understand students' mathematical and personal difficulties and alleviate those difficulties through collaborative partnerships that affirm students' self-identity. Several studies have also demonstrated that teacher-provided opportunities for classroom collaboration and communalism enhance belonging and engagement, especially for students of color (Boykin \& Bailey, 2000; Diekman et al., 2017; Walton \& Brady, 2017). These findings demonstrate how teachers facilitating interconnectedness and social bonds with and among their students can alleviate feelings of alienation and restore students' sense of belonging and mattering while engaging in mathematics. Therefore, creating interpersonal opportunities for belonging is an integral component of the BelongingCentered Instruction framework.

While interpersonal belonging supports are essential, they are not comprehensive nor exhaustive. Green et al. (2016) argued that, because relational interactions for students of color exist within school contexts heavily influenced by politics and culture, the instructional settings within which these interactions materialize likely also affect belonging. In other words, schools and curricula are inextricably bound with the dominant white culture, and teachers risk further undermining student belonging in the math classroom when they deny students of color: 1) the opportunity to see themselves reflected in the curriculum; 2) access to the rigorous mathematical instruction historically denied to them; and 3) space to center and safely explore their culturally-informed mathematical ideas, perspectives, and experiences (Gray et al., 2018; Gutiérrez, 2012). Thus, interpersonal supports for belonging are necessary but insufficient for creating inclusive mathematics environments; teachers must also create opportunities for mathematical belonging through their instructional techniques and pedagogical choices.

Instructional supports for belonging refer to teacher practices that direct instruction toward making meaningful connections between content, the learning process, students' identities, and broader society. A framework that includes instructional supports for belonging is novel compared to those that focus only on interpersonal supports and thus lacks comprehensive empirical support. However, strong theoretical foundations link teachers' instructional and pedagogical choices to students' sense of belonging, especially in mathematics. Gray et al. (2018) argued that mathematical activities that honor and reinforce students' cultural meaning systems through culturally relevant, responsive, and sustaining pedagogies (Gay, 2010; Ladson-Billings, 1995; Paris, 2012) allow students to connect math with their lived realities, thus enhancing the legitimacy of their experiences and their sense of belonging (see also King \& Swartz, 2016). A number of other theoretical and empirical studies have begun to confirm this relationship between centering cultural values through instruction and classroom belonging for students (Boykin \& Ellison, 1995; Dotterer et al., 2009; Rouland et al., 2014). Thus, integrating student culture into the curriculum is a core element of instructional belonging support because centering students' cultural experiences makes mathematics relatable and relevant to their lives.

While Gray et al. (2018) discuss theory and frameworks essential to the Belonging-Centered Instruction model, there are several others that guide the conceptualization of instructional belonging supports. King and Swartz (2016) theorized that the degree to which students of color perceive their intellectual contributions to be valued by teachers also shapes their feelings of belonging. Similarly, certain empirical studies found that belonging for students of color is associated with teachers who promote mastery goals (i.e., prioritizing learning over performance; Dweck, 1986) (Stevens et al., 2007) and provide rigorous expectations and support (i.e., encouraging students to explain and justify their ideas; challenging students mathematically; supporting students through mathematical difficulties) (Beechum, 2020; Stevens et al., 2007). Finally, Kiefer et al. (2015) discovered that teacher support for student autonomy (i.e., student choice with regards to academic tasks) was positively related to their sense of belonging in a multiethnic sample. These studies collectively demonstrate that providing students of color with academic opportunities and resources to thrive in mathematical spaces minimizes feelings of alienation and transforms these spaces to be more inclusive and supportive of students who have been historically marginalized from them. Further, this literature in conjunction with studies of interpersonal belonging supports indicates that Belonging-Centered Instruction can serve as a framework for a more empowering and equitable education for students of color. 


\section{Belonging-Centered Instruction: An Observational Protocol}

Despite the depth and breadth of the belonging literature, there are limitations to how belonging is conceptualized within it. Various studies have conceptualized belonging in disparate ways, including as "school belonging" (Anderman, 2003), "school connectedness" (Anderson-Butcher et al., 2012), "school attachment" (Hill \& Werner, 2006), "peer acceptance" (Linnakyla \& Malin, 2008), "school bonding" (Henry et al., 2005), "school membership" (Smerdon, 2002), "identification with school" (Voelkl, 1996), "sense of social belonging" (Walton \& Cohen, 2011), and "sense of community" (Osterman, 2000), among others. While all circumstantially related (i.e., most focus on socioemotional ties to one's school or agents within it), these various constructs are distinct. Fundamental differences exist in what belonging means according to these constructs, and their conflation has undermined the conceptual clarity of the belonging literature (Gray et al., 2018; Libbey, 2004). Many of these studies also lack an integrated and comprehensive perspective of belonging, and instead focus on discrete aspects of it (Allen et al., 2018; Libbey, 2004; Slaten et al., 2016). The effects of interpersonal relationships, distinct instructional choices, or other factors shaping student belonging are often examined in isolation, which neglects the multidimensionality of belonging and limits the usefulness of these studies for education practitioners (Wallace et al., 2012).

In addition to conceptual concerns related to belonging measures, there are limitations in how these measures are studied. First, much of the empirical work related to student belonging relies on student self-report survey questionnaires (see Allen et al., 2018 for a review of belonging-related research instruments). While some research has incorporated teacher reports of student behavior (e.g., Morrison et al., 2003) or observations of the classroom structure and environment (e.g., Dotterer \& Lowe, 2011), such approaches are rare and only address belonging tangentially, meaning that belonging is not centered in these measures. The absence of observation tools to assess student belonging limits understanding of how it materializes and what practitioners can do to facilitate it. Many existing measures are also domain general (or address belonging at the school level) and are not math specific (Wallace et al., 2012), which neglects the ways math instruction can either facilitate belongingness or alienate students, especially students of color (Martin, 2008, 2013, 2019). Finally, many belonging measures lack an equity and humanization focus (Gray et al., 2018). There is a dearth of studies and instruments that attempt to understand the belonging-related needs of disenfranchised students (Slaten et al., 2016), which perpetuates inequity by neglecting these needs in favor of those that serve the dominant white culture.

In response to these issues, we developed the Belonging-Centered Instruction observational protocol. This protocol allows observers (e.g., researchers, school practitioners) to evaluate the extent to which teachers' actions facilitate belonging in mathematics classrooms through interpersonal and instructional processes. Interpersonal and instructional actions represent two distinct domains of Belonging-Centered Instruction, each of which consists of various subdimensions (see more below) that provide a framework for understanding opportunities for student belonging in a comprehensive and multidimensional way (Wallace et al., 2012). This structure helps observers use the protocol to examine student belonging from an integrated perspective, where they can assess the many, varied, and sometimes disparate sources of belonging holistically. It also encourages researchers to move beyond self-report survey data to understand belonging and allows observers to identify patterns of teacher actions that facilitate opportunities for belonging. Finally, the specific focus on mathematics classrooms serving marginalized students can help us understand how Belonging-Centered Instruction can promote equitable learning experiences for populations that have historically been oppressed in and excluded from mathematics spaces and careers (Bullock, 2017; Diversity in Mathematics Education Center for Teaching \& Learning, 2007), and who have been taught mathematics in dehumanizing ways (Bartolomé, 1994; Gutiérrez, 2018; Martin, 2019; Morton et al., 2020).

\section{The Interpersonal Domain}

As mentioned above, the interpersonal domain of Belonging-Centered Instruction refers to teacher practices that actively facilitate social ties, social comfort, and interconnectedness between members of a classroom. This domain is divided into three distinct subdimensions (Social \& Emotional Bridging, Communal Orientation, and Empathetic Awareness \& Support), each of which embodies the domain's definition in some way.

The first interpersonal subdimension, Social \& Emotional Bridging, describes how teachers can create a positive classroom environment that deconstructs social boundaries between themselves and their students. Here, the teacher shares their humanity (e.g., opinions, likes, stories, personal details) and relates to students in ways that recognize students' humanity (i.e., seeing them as whole people, not just as students). The teacher and students also share emotions and experiences that help each other become "seen" and "known." Teachers can enact Social \& Emotional Bridging through several action indicators, including 1) engaging in social talk and lightheartedness (i.e., using social moments with students to build rapport and uplift relational health); 2) applying cultural competence to build social climate (i.e., drawing on students' cultures to build relational bonds and remaining attentive to cultural nuances in relations with students); 3 ) offering self-disclosures (i.e., information about the teacher that students are unlikely to learn from other sources); 4) exuding 
enthusiasm (i.e., excitement about students or their social or mathematical disclosures); and 5) sharing enthusiasm (i.e., excitement with students over social or mathematical matters). Teachers who engage in these actions facilitate student belonging by dismantling strict teacher-student hierarchies that can alienate students and by encouraging deeper interpersonal relationships between members of the classroom (Allen et al., 2018; Hughes, 2011). This promotes equity by increasing teachers' accessibility and by allowing marginalized students to feel "seen" and "known," as opposed to being reduced to static stereotypes and stigmas (Gutiérrez, 2012).

The second interpersonal subdimension, Communal Orientation, describes how teachers can promote a "we're in this together" orientation with their students. To do so, the teacher supports a climate of communalism (as opposed to individualism) across the social dynamics within the classroom. The teacher also maintains a vested interest in the academic and social growth of the group, and emphasizes the importance of students relying on one another to achieve that growth. Teachers can enact Communal Orientation through several action indicators, including 1) demanding communalism (i.e., prioritizing collective work and communicating its value); 2) teaching interdependence (i.e., providing students with directives on how to work collaboratively); 3) affirming communalism (i.e., praising students for cooperation or interdependence); 4) initiating communal calls and responses (i.e., calls that elicit specific responses from students and that promote class unity); and 5) using communal language (i.e., language that expresses partnership with students or forges a classroom identity). Because many marginalized communities maintain cultural frameworks that are oriented towards communalism or that emphasize interpersonal relationships and collaboration (Boykin et al., 1997; Morton et al., 2020), such teacher actions can promote belonging for students from those groups and prevent further marginalization of students' cultures. Teachers that create space for students to work in communal and collaborative contexts while engaging in mathematics also intentionally honor the cultural values of their marginalized students, which promotes equity through centering students' identities inside the classroom (Gutiérrez, 2012; Morton et al., 2020).

The final interpersonal subdimension, Empathetic Awareness \& Support, describes the teacher's consciousness of individual students' strengths, emotions, physical wellbeing, and resource needs. The teacher is supportive of students' emotional and psychological health, demonstrates that individual students matter in the life of the classroom, and emphasizes students' value regardless of their mathematical ability. Teachers can enact Empathetic Awareness \& Support through several action indicators, including 1) acknowledging student difficulties (i.e., verbally recognizing students' academic, emotional, and physical challenges or needs); 2) addressing barriers to student wellbeing (i.e., providing focused time and attention to alleviate anything impinging on students' wellbeing); 3) providing personalized affirmations (i.e., praise tailored to individual students that recognizes their unique strengths, accomplishments, or contributions); and 4) creating roles that allow individual student contributions (i.e., utilizing student roles to provide opportunities for students to take pride in what they can uniquely offer). Teachers that empathetically address student needs and emphasize students' value in these ways can promote belonging by mitigating the alienation many marginalized students feel in classrooms where their needs are often deprioritized in favor of curriculum pacing and content coverage (Maloney \& Matthews, 2020). Such actions can also promote equity by elevating marginalized students' sense of personal mattering and by supporting their psychological and emotional health (Kozlowski \& Si, 2019; Maloney \& Matthews, 2020).

\section{The Instructional Domain}

The instructional domain of Belonging-Centered Instruction refers to teacher practices that create opportunities for students to make meaningful connections between course content, learning processes, their identities, and broader society. This domain is divided into four distinct subdimensions (Safety to Be Wrong, De-centering Teacher Authority, Mathematics to Know Myself \& My World, and High Standards \& Rigorous Support).

The first instructional subdimension, Safety to Be Wrong, pertains to how the teacher creates a space where students do not feel stigmatized for wrongness or needing support in the classroom. The teacher disarms and normalizes wrongness, and creates opportunities for students to resist judging their own mathematical belongingness simply based on correctness. Mastery, effort, and strategy are celebrated over performance and talent. Teachers can enact Safety to Be Wrong through several action indicators, including 1) normalizing difficulties (i.e., showing that wrongness is a normal and expected part of learning); 2) creating space for questions (i.e., seeking out questions from students to create an environment safe for uncertainty and learning); 3) providing task-focused encouragement (i.e., disarming self-doubt and encouraging persistence through difficult mathematical tasks); 4) celebrating mastery-focused behavior (i.e., praising students' efforts and strategies as a way to achieve mastery), and 5) modeling mistakes for learning (i.e., using teacher or student mistakes as opportunities for learning). Teachers that prioritize mastery and effort over performance and correctness in these ways can facilitate students' sense of belonging in mathematics classrooms (Stevens et al., 2007). Such actions also destigmatize and remove the threat associated with wrongness that many marginalized students encounter in mathematics, thus inviting more equitable participation (Gutiérrez, 2012; Kozlowski \& Si, 2019). 
The second instructional subdimension, De-centering Teacher Authority, describes how the teacher communicates that students' mathematical methods and ideas have real value and worth. The teacher positions students as knowledge authorities in mathematics and conveys that students' intellectual contributions matter in the life of the classroom. Teachers can enact De-centering Teacher Authority through several action indicators, including 1) engaging students' expertise (i.e., soliciting students' unique conceptual insights and deep expertise); 2) honoring multiple ways of knowing and showing (i.e., affirming students' unique mathematical ideas that demonstrate multiple pathways to solving a problem); 3) providing opportunities for agency (i.e., sharing power with students and following their leads regarding classroom instruction and activities); and 4) inviting feedback on instruction (i.e., asking for feedback on the effectiveness of the math instruction and activities). Because many marginalized students feel alienated in mathematics classrooms where the knowledge and expertise that they bring with them are ignored, valuing students' insights and autonomy in these ways can promote a sense of belonging (Kiefer et al., 2015; King \& Swartz, 2016). A de-centered teaching approach also promotes equity by empowering marginalized students to claim agency as mathematical learners and thinkers and by elevating their voices in the classroom (Gutiérrez, 2012).

The third instructional subdimension, Mathematics to Know Myself \& My World, reflects how the teacher can create opportunities for students to use math to empower cultural identity, understand the world, and critique their social context. Teachers can enact Mathematics to Know Myself \& My World through several action indicators, including 1) elaborating on mathematical relevance (i.e., making space for discussions and activities to understand the deeper relevance and meaning of math concepts); 2) engaging in culturally relevant and responsive instruction (i.e., instruction that is responsive to, learns from, or integrates students' cultural preferences or meaning systems); 3) providing teacher-generated real-world connections (i.e., applications of math concepts to real-world issues and topics); 4) drawing on student-generated real-world connections (i.e., encouraging or creating space for students to apply math concepts to real-world issues and topics); and 5) using mathematics to help students become informed citizens (i.e., providing students with opportunities to use math to empower their sense of cultural identity and critique social injustice). Such actions can promote students' sense of belonging by buffering against the alienation students feel when mathematics is disconnected from their social and cultural realities (Gray et al., 2018). This approach can also promote equity by allowing students, especially those who are marginalized, to see themselves reflected in the mathematics curriculum and by highlighting math as a tool useful for navigating the world and combating social injustice (Bartell, 2013; Gutiérrez, 2012).

The final instructional subdimension, High Standards \& Rigorous Support, describes how the teacher can communicate high standards for students while also providing structured support to help students achieve these standards. Here, the teacher implicitly or explicitly expects consistent mathematical effort and high-level performance. While maintaining high standards and offering support, the teacher also instills confidence or shares strategy in order to cultivate the belief within students that they can fulfill the high expectations. Teachers can enact High Standards \& Rigorous Support through several action indicators, including 1) pressing on the process (i.e., encouraging students to use appropriate terminology and mathematical procedures, and understand their importance); 2) affirming and pushing (i.e., praising students' achievements in the problem-solving process while stretching students to improve what they have already done); 3 ) providing personalized scaffolding (i.e., prolonged scaffolding that is specifically tailored to a student's mathematical needs); and 4) prepping and empowering students to achieve (i.e., providing strategy and encouragement to empower and prepare students for important achievement goals). Because marginalized students are often held to lower mathematical standards and receive less rigorous instruction, communicating high expectations in these ways can enhance their feelings of belongingness by indicating their potential for strong mathematical achievement (Beechum, 2020; Stevens et al., 2007). Providing students with the skills, strategies, and support to meet these expectations can also promote equity by providing students with the means to thrive mathematically (Gutiérrez, 2012).

\section{Development of the Belonging-Centered Instruction Observational Protocol}

In developing the Belonging-Centered Instruction observational protocol, the research team worked to avoid the common limitations of survey and observational measures related to belonging. Many of the existing measures are topdown (i.e., etic) as opposed to bottom-up (i.e., emic), which grounds them within the logic of the researchers more than the research participants (Adair \& Pastori, 2011; Headland et al., 1990). Because scholarship in education has been dominated by white researchers studying white students, most existing studies have been disconnected from the lived experiences and perspectives of students of color. This limitation has resulted in the development of measures that fail to adequately honor or capture students' experiences and perspectives, especially as they relate to their sense of mattering and belonging in the classroom. The Belonging-Centered Instruction observational protocol is unique in that its development was derived from the convergence of three sources (see Figure 01), the first two of which explicitly integrate students' lived experiences in mathematics classrooms: 1) qualitative analysis of themes from longitudinal interviews from Black and Latinx students over 
four years $(n=37) ; 2)$ a grounded theory process of open coding video-recorded classroom observations and iteratively refining the saturated action indicators and cultivated subdimensions; and 3) previous theory and research on belonging.

\section{Figure 01}

The Three Sources of the Belonging-Centered Instruction Observational Protocol

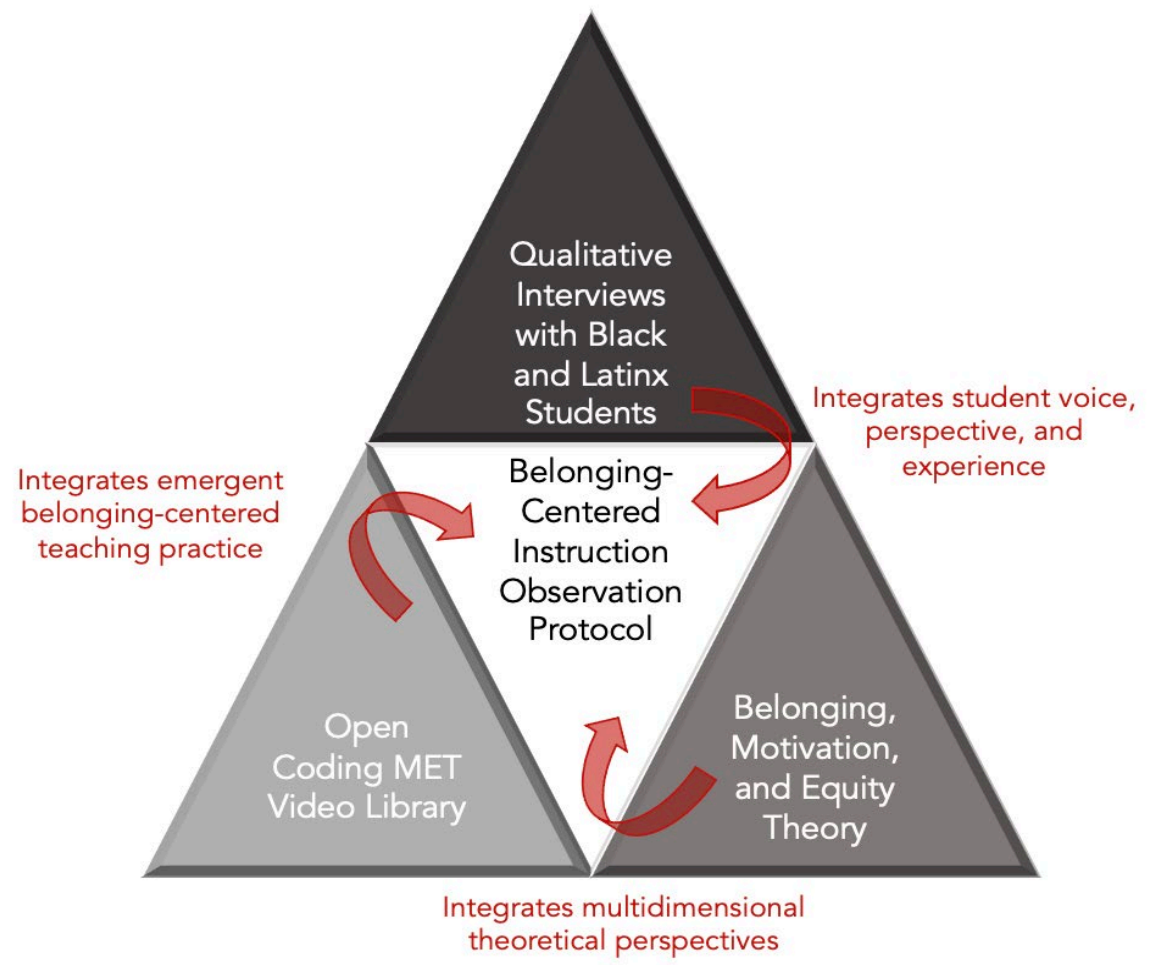

\section{Qualitative Interviews:}

The qualitative interviews used to develop this protocol were drawn from a previous longitudinal study (Matthews, 2018) that investigated how urban adolescents negotiated the meaning of mathematics in school and in their lives more broadly. The sample from that larger study included 419 fifth- through twelfth-grade adolescents (53\% women; 56\% Black, 27\% Latinx, 3\% White) from a large city in New Jersey (52\% Black, 33.8\% Latinx; median income was \$35,659; U.S. Census Bureau, 2010). Data was collected each year between the 2014-2015 and 2018-2019 school years. Of this original sample, 37 students were selected to participate in interviews annually over the course of four years. Interviewees were selected using maximum variation sampling (Miles \& Huberman, 1994), where the subsample reflected a balance of girls and boys; Black and Latinx students; and low, moderate, and high levels of engagement within math class. Interviewers were trained to use a semi-structured interview protocol that aimed to capture the students' math beliefs, experiences in the math classroom, and perspectives on race and racial identity, as well as how students made meaning of and negotiated these beliefs and experiences. Interviews were conducted with each student once per year for four years during the spring terms, typically within students' math classrooms after school. Each time data was collected, the principal investigator (PI) and a team of research assistants coded and analyzed the interview transcripts to identify and organize students' perspectives and experiences related to various topics, including their sense of belonging in mathematics.

From these interviews, the research team identified eight broad themes related to interviewees' perceptions of how their teachers cultivated opportunities for belonging in the math classroom. Four of these themes were categorized as interpersonal belonging supports, and the remaining four were categorized as instructional belonging supports (Gray et al., 2018). In the interpersonal domain, one identified theme was "Teacher Empathetic Care," which reflected teachers' authentic expressions of identifying with the challenges of their students and prioritizing students' wellbeing above their own. This theme was most clearly illustrated in the following segment:

Like something always aggravates me. I get aggravated fast ... I'll be walking to the class, [the teacher] will stop me outside. She be like, "You okay?" I just be like [shrugs]... She just be like, "I'll talk to you after class"... Then she talk to me after class. She ask me, am I okay? Do I need somebody to talk to? Like what's bothering me?... It 
makes me feel like I have somebody in the school, not just at home, that I could talk to... Like somebody that's there for me. [Imani ${ }^{1}, 10^{\text {th }}$ grade student, woman, African American].

Here, the teacher demonstrated "Empathetic Care" through her consciousness of the student's emotional difficulties and by offering authentic support, which facilitated feelings of belongingness within the student.

In the instructional domain, one identified theme was "Mathematics to Become a Critical Citizen," which reflected how teachers could provide opportunities for students to use math to empower their sense of cultural identity, critique their social world and social injustice, and challenge the status quo. This theme was most clear in the following segment:

[The way math is taught], it's kind of pretty much, you do this, you do this, there is no creativity, it's just this... It's just you learn this formula, you do this formula, that's it... It makes us stay in the same place. Like literally every single achievement was because someone decided that they didn't like the way something was going, so you're gonna change it to be more creative... Like let's say one day you're gonna get a project to figure out a way math can help someone... That's how new math formulas could be taught. [Hakeem, $9^{\text {th }}$ grade student, man, African American].

Here, the student expressed feeling alienated and limited in his current math classroom, and he conceptualized a form of teaching (i.e., "Mathematics to Become a Critical Citizen") that mitigated those feelings by centering the content within students' interests and experiences.

These eight themes provided the general framework for the Belonging-Centered Instruction observational protocol and general patterns to compare and negotiate during open classroom coding.

\section{Open Classroom Video Coding:}

Following the interview analysis, the PI formulated a new team of interdisciplinary scholars and students to refine, modify, and synthesize the interview themes by integrating new insights and themes that emerged through an open coding process of video recorded mathematics classrooms. Open coding is a grounded theory method of attaching words and short phrases (i.e., codes) to observed data to capture its essence, attributes, and elements (Saldaña, 2009; Strauss \& Corbin, 1990). This method is typically applied to interview text; however, for the purpose of this project, the research team applied it to discourse and events present in video recordings of mathematics classrooms. The team observed mathematics teachers' classrooms, grades six through eight, by accessing pre-recorded videos located in the Measures of Effective Teaching Longitudinal database (MET-LDB). The MET-LDB includes data collected from a partnership between researchers and schools from 2009 to 2013 in the following regions and urban school districts: Charlotte-Mecklenburg County Schools (North Carolina), Dallas Independent School District (Texas), Denver Public Schools (Colorado), Hillsborough County Public Schools (Florida), Memphis City Schools (Tennessee), and the New York City Department of Education (New York).

The coding process occurred in three phases, each emphasizing the teacher behaviors that seemed to create opportunities for belonging in their mathematics classrooms. Table 01 includes a description of each phase.

\footnotetext{
${ }^{1}$ All names provided are pseudonyms to protect students' anonymity.
} 
Table 01

Open Video Coding Process

\begin{tabular}{lll}
\hline Phase & Timeframe & Description \\
\hline Codebook development & $\begin{array}{l}\text { October 2019 }- \\
\text { January 2020 }\end{array}$ & $\begin{array}{l}\text { Researchers employed initial coding and simultaneous coding to } \\
\text { identify teacher actions (i.e., codes) that created opportunities for } \\
\text { student belonging. These codes were applied iteratively to } \\
\text { observation videos to reveal patterns in video data and develop an } \\
\text { initial codebook. }\end{array}$ \\
\hline Codebook refinement & $\begin{array}{l}\text { January 2020 - } \\
\text { April 2020 }\end{array}$ & $\begin{array}{l}\text { Researchers collaboratively clarified conceptual distinctions } \\
\text { between codes by discussing how events and discourse that } \\
\text { reflected these codes compared within and across classrooms. The } \\
\text { codes and their descriptions were modified and refined } \\
\text { accordingly to ensure they could be applied reliably and to } \\
\text { maintain their conceptual integrity. }\end{array}$ \\
\hline
\end{tabular}

$\begin{array}{ll}\text { Code sorting and } & \text { April } 2020- \\ \text { theme refinement } & \text { September 2020 }\end{array}$

Researchers examined how codes related to each other, the broader themes that emerged from the interview data, and previous theory related to belonging and learning equity. Themes were refined, removed, collapsed, parsed, or generated to be inclusive of the codes that materialized in the open video coding process. Codes were also refined during this process. The final solidified themes were renamed "subdimensions" and their respective codes "action indicators" within each subdimension.

$\begin{array}{ll}\begin{array}{l}\text { BCI scoring protocol } \\ \text { development }\end{array} & \text { July } 2020- \\ \text { October } 2020\end{array}$

Researchers constructed several models for observational scoring of BCI and applied them to the same set of videos as well a new set of videos not previously viewed. After examining advantages and disadvantages for each, the most adaptive version was selected, and it was tested and revised repeatedly until reaching adequate inter-rater reliability of scoring.

\section{Codebook Development.}

During the first phase, the team analyzed videos from the MET-LDB and took notes on verbal and nonverbal interactions teachers had with their students while documenting the timestamps in which the events occurred during the video (for more on analyzing video-recorded data, see Erickson, 2006). The team employed initial coding to capture first impressions of how these interactions related to belonging and then utilized simultaneous coding (i.e., applying two or more codes to the same data) (Saldaña, 2009) to refine the initial impressions and generate further understanding of how teachers' behaviors actually facilitated belonging. During the simultaneous coding process, the team used both process codes (i.e., research-generated words or phrases) and in vivo codes (i.e., verbatim words or phrases of the participants) to capture and label belonging-relevant teacher actions from the MET-LDB videos (Saldaña, 2009). In addition to identifying new codes that materialized, the first phase of coding included an ongoing process of codifying, which refers to iteratively applying codes to group data together based on similar characteristics (Saldaña, 2009). This cumulative process resulted in an exhaustive codebook that included code names and descriptions as well as illustrative examples from specific videos for each code.

\section{Codebook Refinement.}

The second phase of the coding process focused on refining the codebook and clarifying conceptual distinctions between codes. Using video analysis software to analyze a new set of classroom videos from the MET-LDB, the team worked to ensure the conceptual integrity of each code by comparing codes within and across the videos. Throughout this process, the team also consistently reviewed the codebook by examining each code individually as it related to the others. This process allowed the team to identify discrepancies between codes; refine and condense code names, descriptions, and illustrative examples; and ensure the codes could be applied reliably across multiple and unique contexts. Once the codebook was refined, researchers worked to further develop the themes from the qualitative interviews and existing research using the codes generated during the open coding process. 


\section{Code Sorting and Theme Refinement.}

During the third phase of the open coding process, the team transitioned away from considering codes individually and instead examined how the codes related to each other and to the eight broader themes that emerged from the qualitative interviews. Through code-grouping, the team collaboratively decided which codes aligned with each theme (e.g., the Normalizing Difficulties code aligned with Safety to Be Wrong theme), removed themes where codes did not fit (e.g., Personalized vs. Rote Scaffolding, Teachers without Borders), or modified themes to be more inclusive of the codes present (e.g., Teacher Empathetic Care became Empathetic Awareness and Support to be more inclusive of the ways that teachers might express empathetic and caring support). Once the team solidified each theme and its respective codes, themes were renamed "subdimensions," and their codes were renamed the "action indicators" of each subdimension. These subdimensions and their respective action indicators formed the foundation of the Belonging-Centered Instruction observational protocol.

\section{Belonging-Centered Instruction Observational Protocol Development.}

After solidifying the subdimensions and their respective action indicators, researchers constructed the BelongingCentered Instruction observational protocol. The team reviewed several established observational measures used to evaluate the quality of various classroom interactions. Each team member then constructed their own prospective model of the Belonging-Centered Instruction observational protocol, and researchers met several times to analyze the strengths and limitations of each model. Three new models were then created that integrated the identified strengths and minimized limitations. Researchers applied these three synthesized models of the scoring protocol to the same video, discussed the advantages and disadvantages of each model, and then selected and revised the most adaptive version. This version was tested and revised repeatedly until the research team reached adequate interrater reliability.

\section{Belonging, Motivation, and Equity Theory:}

Finally, the development of the Belonging-Centered Instruction observational protocol was also guided by existing theoretical and empirical research related to belonging. Gray et al. (2018) helped the research team conceptualize multiple domains of teacher belonging supports (i.e., interpersonal and instructional) and provided a multidimensional perspective on this approach to belonging, which is lacking in much of the existing literature (Allen et al., 2018; Libbey, 2004; Slaten et al., 2016). Additional research on belonging informed the Belonging-Centered Instruction subdimensions, specifically by helping the research team refine the meaning and implications of constructs within the interpersonal subdimensions (Allen et al., 2018; Boykin \& Bailey, 2000; Diekman et al., 2017; Hughes, 2011; Maloney \& Matthews, 2020; Walton \& Brady, 2017) and the instructional subdimensions (Beechum, 2020; Boykin \& Ellison, 1995; Dotterer et al., 2009; King \& Swartz, 2016; Rouland et al., 2014; Stevens et al., 2007) (see more on the various subdimensions in the section, "Belonging-Centered Instruction: An Observational Protocol," above).

The Belonging-Centered Instructional observational protocol was also grounded in theoretical frameworks that confirm the need for belonging within mathematics classrooms, especially for students of color, such as stage-environment fit theory (Eccles \& Midgley, 1989) and self-determination theory (Deci et al., 1991). According to stage-environment fit theory, interpersonal and instructional belonging supports can produce increased wellbeing and motivation by satisfying the psychological need to belong (Eccles \& Midgley, 1989; Eccles et al., 1993; Gillen-O’Neel \& Fuligni, 2013). Similarly, selfdetermination theory suggests that when students' needs for relatedness (i.e., belongingness) are fulfilled by interpersonal and instructional belonging supports, students may better internalize important academic values and develop a stronger sense of autonomy and intrinsic motivation (Deci et al., 1991; Ryan, 1995). These guiding theories demonstrate the potential of Belonging-Centered Instruction to support equity and empowerment for marginalized students of color by outlining clear socioemotional and academic benefits for student belonging in mathematics classrooms.

Finally, research related to learning equity in mathematics education (Gutiérrez, 2012; Kozlowski \& Si, 2019; Martin, 2008, 2013, 2015, 2019; Martin et al., 2010), informed how the Belonging-Centered Instruction observational protocol was developed, refined, and framed. Gutiérrez (2012) conceptualized a model for mathematics learning equity based on four distinct but related dimensions: access (i.e., the accessibility of resources needed to thrive in mathematics), achievement (i.e., positive student outcomes in math class that facilitate long-term academic success), identity (i.e., students being reflected in the curriculum and understanding how math can be used to analyze the world), and power (i.e., opportunities to transform social relations and the broader world using math and within mathematical spaces). However, barriers to belonging compromise the fulfillment of each of these dimensions of equity. For example, when Black and Latinx students are denied access and achievement through discriminatory discipline and tracking practices (Musu-Gillette et al., 2016; Oakes, 2005; Scott et al., 2017), and when they are denied identity and empowerment through "colorblind" instruction that perpetuates white supremacy (Battey, 2013; Martin, 2008, 2013, 2015), not only is their sense of belonging impeded, but they are also denied an equitable mathematics education (Gutiérrez, 2012). Therefore, Gutiérrez's model is reflected in 
the conceptualization of Belonging-Centered Instruction, which is defined as teachers' provision of opportunities for active inclusion, achievement, identification, and empowerment through interpersonal interactions and instructional techniques.

Altogether, this research shaped how the research team considered the data and understood the findings throughout the various stages of the Belonging-Centered Instruction observational protocol's development, and its synthesis facilitated the development of an integrated, multidimensional, and inclusive conceptualization of belonging for marginalized students in mathematics classrooms.

\section{Video Scoring Sampling Criteria}

Once the protocol was completed, researchers tested the reliability and predictive validity of the protocol and our coders by using it to score middle grades mathematics classroom videos from the MET-LDB. The MET-LDB includes a large longitudinal database of observational videos; survey data from students, teachers, and school leaders; and standardized achievement scores. The process for selecting the subsample of instructional videos that were used to validate the Belonging-Centered Instruction observational protocol is outlined below.

\section{Inclusion and Exclusion Criteria}

As described in Figure 02, 24,659 instructional videos were available in the MET-LDB. To select a subset of videos for evaluating and validating the protocol, the research team first focused specifically on classroom videos recorded in the second year (Year 2) of this partnership $(n=5,373)$. Partnering schools in Year 2 agreed to randomly assign students to mathematics classrooms. To randomize assignment, MET-LDB project leaders created "exchange groups" of teachers who taught the same subject in the same grade and were certified to teach all common classes. They also created randomization blocks to assign students to classrooms, which ensured variability of students (i.e., students of all skill levels) across all classrooms within schools. It should be noted that not all Year 2 schools participated in randomization, either because the school's teachers did not participate in Year 2 of the study or their classrooms could not be randomized due to scheduling conflicts. Classrooms that did not meet the randomization criterion for Year 2 were excluded from the selection and analysis. Focusing on classroom where students were randomized served as a starting point for further screening. It also enabled the classrooms to be as close to a statistically equivalent and unbiased baseline as possible, which allowed a more accurate assessment of whether Belonging-Centered Instruction accounted for positive student outcomes (versus other idiosyncratic features of the class if there was no random assignment) (Coolican, 2018; Remler \& Van Ryzin, 2010).

Because this research focused on middle school mathematics classrooms predominantly serving students of color, we developed exclusion criteria to eliminate videos of 1) classrooms serving students below grade six or above grade eight, 2) English and Biology classrooms, and 3) classrooms that were predominantly white. To meet the third criterion, a majority percentage cutoff was employed such that only classrooms in which at least $50 \%$ of students were non-white students of color (i.e., Black/African American, Hispanic/Latinx, Asian/Asian American, multiracial) were retained in the dataset. This criterion placed students of color as the numerical majority and the focus of classroom activities. As a result of these criteria, 952 videos were excluded for serving students in classrooms below or beyond grades six through eight, 2,429 videos were excluded for focusing on content unrelated to mathematics, and 377 videos were excluded for having a predominantly-white student population. 
Figure 02

Map of Video Sampling Based on Inclusion and Exclusion Criteria

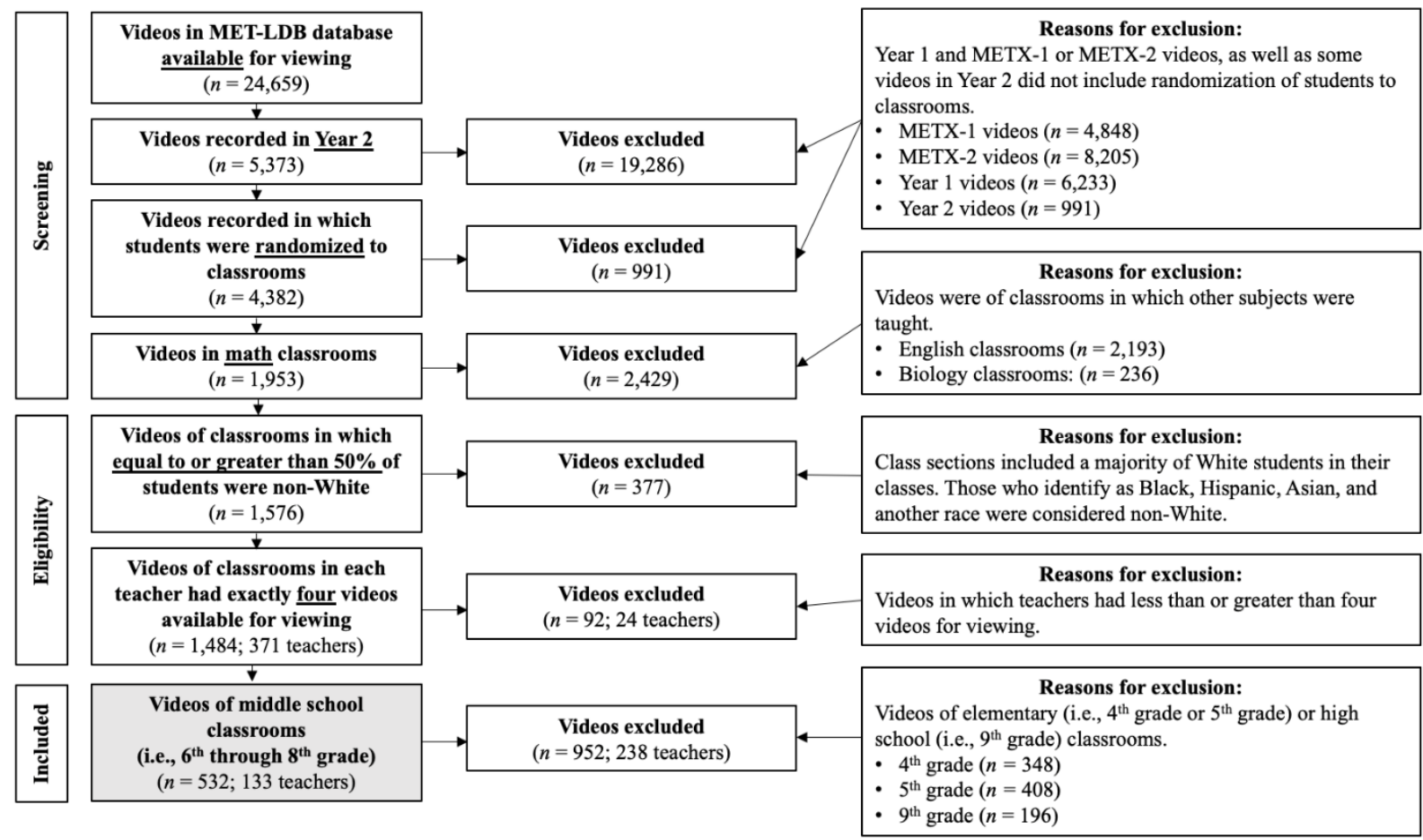

One unique criterion for sampling was selecting only teachers who complied with study protocol by recording exactly four classroom videos over the course of the school year. It is not clear why some teachers recorded fewer or more than four videos, but we considered that these unknown variables might have biased the sampling strategy in some way. Therefore, the research team excluded videos from teachers who did not follow the study protocol $(n=92)$. Considering these inclusion and exclusion criteria, this yielded a final sample size of 532 classroom videos collected by 133 teachers.

\section{Video Scoring}

After selecting the final sample of classroom videos for this project, the team began scoring them using the Belonging-Centered Instruction observational protocol. That scoring process is outlined below.

\section{Video Cycles}

For each of the 133 teachers remaining after applying the inclusion and exclusion criterion, the first three consecutive classroom videos recorded from Year 2 of the MET-LDB project were scored. This resulted in a final sample of 399 classroom videos that we scored for reliability and predictive analyses across all 133 teachers. Because classroom videos varied in time length, the first 45 minutes of each video was observed and scored. This time period reflected the average length of classroom videos and the typical length of a middle school math lesson. Scoring multiple videos per teacher allowed the research team to examine within-teacher consistency. Given the dynamic nature of teaching in middle grades classrooms, any one class session may not fully capture the variety, breadth, and quality of teacher actions and classroom climate that observing multiple class sessions would afford.

\section{Independent Scorers}

Each video cycle was scored by at least two independent, trained scorers. Scorers were members of the research team who participated in the development of the Belonging-Centered Instruction observational protocol and/or who were trained for several weeks to use the protocol. Pairs of scorers watched each video independently and met weekly to discuss disagreements in scoring.

\section{Scoring Protocol}

Each teacher received 16 scores for each classroom video observed. Fourteen scores were provided to evaluate the subdimensions in the instructional and interpersonal domains, and two scores were provided to evaluate threats to student belonging (as described in the Belonging-Centered Instruction observational protocol; see pg. 25). For each subdimension in the instructional and interpersonal domains, teachers received two distinct scores: one for the subdimension's quality, and one for the subdimension's variation. A subdimension's quality describes the degree to which a teacher's actions 
fulfilled that subdimension's description during the observation cycle. A subdimension's variation describes the range and quantity of specific action indicators that were present throughout the observation cycle.

The instructional domain (i.e., instructional belonging supports), which encompassed educator practices that helped students make meaningful connections between course content, the learning process, their identities, and broader society, was comprised of eight distinct scores. Each of the four subdimensions (Safety to Be Wrong, De-centering Teacher Authority, Mathematics to Know Myself \& My World, High Standards \& Rigorous Support) was scored on a Likert scale from one to six for both quality and variation, resulting in eight scores total. The interpersonal domain (i.e., interpersonal belonging supports), which encompassed educator practices that actively facilitated social ties, social comfort, and interconnectedness between members of the classroom, was comprised of six distinct scores. Each of the three subdimensions (Social \& Emotional Bridging, Communal Orientation, Empathetic Awareness \& Support) was likewise scored on a Likert scale from one to six for both quality and variation, resulting in six scores total.

Finally, two scores captured moments in which teachers posed Threats to Belonging, or used practices that explicitly undermined or posed a threat to students' belonging. Each classroom video received two scores from zero to negative two for absent, moderate, or overt actions that countered the seven previously described subdimensions in the interpersonal and/or instructional domain. In other words, classroom videos were given two separate scores ranging from zero (absent) to negative two (overt), one score for interpersonal threats to belonging and the other for instructional threats to belonging.

\section{Interrater Reliability}

For each classroom video scored, an overall rating of teacher support in the interpersonal and instructional domains was calculated by averaging ratings across individual categories for each domain. Following previously established guidelines for assessing reliability using intraclass correlation coefficients (ICC) (Koo \& Li, 2016), ICC estimates between a value of .50 and .75 reflect moderate reliability, and estimates with a value greater than .75 reflect strong reliability. Based on a one-way random effects model, both the quality and the variation values for the interpersonal and instructional domains fell within these ranges: ICC for BCI Instructional Support (Variation) = .68; ICC for BCI Instructional Support (Quality) $=.70$; ICC for BCI Interpersonal Support (Variation) = .75; ICC for BCI Interpersonal Support (Quality) = .79. The quality and variation ICC values for the various interpersonal and instructional domains fell within this range as well (see Table 02 below).

Table 02

ICC Values for BCI Subdimensions

\begin{tabular}{lc}
\hline \multicolumn{1}{c}{ Subdimension } & ICC Value \\
\hline Safety to Be Wrong (Quality) & .63 \\
Safety to Be Wrong (Variation) & .61 \\
Decentering Teacher Authority (Quality) & .67 \\
Decentering Teacher Authority (Variation) & .67 \\
Mathematics to Know (Quality) & .51 \\
Mathematics to Know (Variation) & .54 \\
High Standards \& Rigorous Support (Quality) & .52 \\
High Standards \& Rigorous Support (Variation) & .53 \\
Social \& Emotional Bridging (Quality) & .82 \\
Social \& Emotional Bridging (Variation) & .78 \\
Communal Orientation (Quality) & .72 \\
Communal Orientation (Variation) & .69 \\
Empathetic Awareness \& Support (Quality) & .68 \\
Empathetic Awareness \& Support (Variation) & .66 \\
\hline
\end{tabular}

\section{Use Cases}

The research team envisioned multiple contexts to which the BCI protocol could be applied (i.e., use cases). In this study, the researchers identified two distinct use cases that would highlight the various practices and outcomes associated with belonging-centered teaching. In Use Case 01, the researchers used the BCI protocol to predict various student outcomes that were expected to materialize when Interpersonal and Instructional belonging supports were enacted in mathematics classrooms. In Use Case 02, the researchers paired the BCI protocol with state space grids (SSGs) to document the dynamic interplay of Interpersonal and Instructional subdimensions across classroom periods. These two use cases will be further discussed in the following. 


\section{Use Case 01: Using the BCI Protocol to Predict Student Outcomes Design}

For Use Case 01, the research team evaluated the predictive validity of the BCI Observation Protocol. The researchers hypothesized teachers' domain (i.e., Interpersonal and Instructional belonging supports) and subdimension (e.g., Safety to Be Wrong, Social \& Emotional Bridging) scores would be positively associated with various student outcomes related to student belonging and achievement. Further, the researchers hypothesized that certain school-level factors (e.g., administrative support) would be positively associated with teachers' subdimension and domain scores. In all analyses, domain and subdimension scores refer to their respective Quality scores (i.e., not Variation scores; predictive analyses using Variation scores are forthcoming). ${ }^{2}$ These predictive analyses were guided by the following research questions.

RQ1: Do the BCI subdimension and domain scores predict student engagement in middle school mathematics classrooms?

RQ2: Do the BCI subdimension and domain scores predict students' self-reported mathematical agency in middle school mathematics classrooms?

RQ3: Do the BCI subdimension and domain scores predict students' year-end mathematics achievement?

RQ4: Do teachers' self-reported perceptions of the quality of their administrators' support \& feedback predict their $\mathrm{BCI}$ subdimension and domain scores?

\section{Measures}

BCI Subdimension Scores. BCI subdimension scores reflected the degree to which teachers' instructional and interpersonal interactions with students fulfilled the respective subdimensions' descriptions (see pg. 26) over a single classroom episode. The seven subdimensions scored for each video included Social \& Emotional Bridging, Communal Orientation, Empathetic Awareness \& Support, Safety to Be Wrong, Decentering Teacher Authority, Mathematics to Know Myself \& My World, and High Standards \& Rigorous Support. Two coders scored each subdimension for all teachers $(N=$ 133) based on three of their video-recorded classroom lessons. The scores from the two coders were then aggregated for each subdimension, resulting in each teacher receiving one score for each of the seven subdimensions.

BCI Domain Scores. BCI domain scores reflected the degree to which teachers' instructional and interpersonal interactions with students fulfilled the respective domains' definitions (see pg. 6). Teachers' aggregated subdimension scores were averaged to generate one Interpersonal score and one Instructional score for each teacher. The Interpersonal scores were the average of the Social \& Emotional Bridging, Communal Orientation, and Empathetic Awareness \& Support subdimension scores. The Interpersonal scores were the average of the Safety to Be Wrong, Decentering Teacher Authority, Mathematics to Know Myself \& My World, and High Standards \& Rigorous Support subdimension scores.

Student Engagement. Student engagement was assessed through two distinct measures, one external and one student self-reported. First, researchers used the Student Engagement measure from the Classroom Assessment Scoring System- Secondary (CLASS-S), an observational instrument that assesses multiple subdimensions of teaching that are associated with student achievement (Pianta et al., 2012). This measure evaluated "the degree to which all students in the class are focused and participating in the learning activity facilitated by the teacher" (Pianta et al., 2012, p. 99). Relevant student behaviors included volunteering, sharing ideas, and manipulating materials. MET researchers used the CLASS-S to score classroom videos from teachers in the broader MET project using a 7-point scale $(1=$ Low to $7=$ High $)$. Available Student Engagement scores for classrooms in the current project's sample were aggregated at the teacher level, where each teacher received a composite Student Engagement score.

Student engagement was also assessed using the Captivate measure from the Tripod 7C's Framework of Effective Teaching survey (Ferguson, 2012). This survey evaluated students' perceptions related to their teacher's enactment of seven essential elements of instructional practice (e.g., Care, Clarify, Challenge), and was part of the broader Student Perceptions Survey (SPS) that was administered to all students consented to participate in the MET project during the spring of the 2010-2011 academic year. The Captivate measure specifically assessed students' self-reported perceptions of their teachers' ability to cultivate curiosity, interest, and engagement. Students rated the four Captivate items (e.g., My teacher makes

\footnotetext{
${ }^{2}$ Quality again refers to the degree to which the teachers' actions fulfilled the description of the domain or subdimension throughout the observation cycle. Variation instead refers to the range and/or quantity of different teacher actions (i.e., action indicators) that were present throughout the observation cycle.
} 
learning enjoyable $)$ on a five-point Likert scale $(1=$ Totally Untrue to $5=$ Totally True; $\alpha=.94)$. Student responses were aggregated to the teacher level to create composite scores for each item for each teacher, which were aggregated again to create a single composite Captivate score for each teacher.

Student Mathematical Agency. Students' mathematical agency was assessed through the Confer measure from the Tripod 7C's Framework of Effective Teaching Survey (Ferguson, 2012), which was also part of the SPS. The Confer measure specifically evaluated students' self-reported perceptions of their ability to direct classroom activities, share ideas, and feel valued and respected within the mathematics classroom. Students rated the five Confer items (e.g., My teacher respects my ideas and suggestions $)$ on a five-point Likert scale $(1=$ Totally Untrue to $5=$ Totally True; $\alpha=.88)$. Student responses were aggregated to the teacher level to create composite scores for each item for each teacher, which were aggregated again to create a single composite Confer score for each teacher.

Student Year-End Mathematics Achievement. Students' year-end mathematics achievement was assessed by the Balanced Assessment in Mathematics (BAM). The BAM is an open-ended standardized assessment that evaluates students' abilities related to several categories of mathematical thinking (e.g., inferring/drawing conclusions, modeling/formulating problems). MET researchers administered three different versions of the BAM to students to ensure generalizability of the results. The three versions of the BAM included four or five unique tasks. The categories of mathematical thinking being evaluated by the test were scored for each task on a four-point scale $(1=$ Attribute Not Present to $4=$ Attribute Predominantly Present). Students completed the BAM in the final two months of the 2010-2011 academic year (i.e., after video recordings of classrooms were completed).

Administrators' Support for \& Feedback to Teachers. Teachers' self-reported perceptions of the quality of support and feedback that they received from school administrators were measured through the MET teacher survey, which was distributed after the end of the 2010-2011 school year. This survey evaluated teachers' perceptions related to various administration-level practices and competencies. Of particular interest to the research team were survey items that measured teachers' perceptions of 1) supervisors providing feedback on teachers' instruction, 2) supervisors monitoring teaching methods and performance, 3) principals demonstrating competence at performing their responsibilities, 4) principals demonstrating integrity in the workplace, 5) principals treating staff fairly, and 6) principals being helpful to staff. Each of these practices/competencies was measured by two to three unique survey items, which were combined into composite variables after testing for internal reliability and statistical fit within a confirmatory factor model. This yielded six composite variables that represented teachers' perceptions of various administration-level factors that could affect their practice.

\section{Results}

Prior to addressing the research questions, the team evaluated bivariate correlations between the scores of the two domains and seven subdimensions. To assess RQ1-3, the research team used Stata 16 to conduct several multiple regression analyses, where the domain and subdimension scores were modeled as independent variables, and student outcomes (e.g., student engagement, mathematical agency, achievement) were modeled as dependent variables. Each outcome was assessed at the classroom level (i.e., BCI scores were used to predict aggregated classroom-level outcomes). In all of these multiple regression analyses, the team used dummy variables to control for school district given the potential variation of BCI practices across geographical regions and districts. The students were also randomized into classrooms, which served as another control by minimizing statistically significant differences between classrooms and districts (Remler \& Van Ryzin, 2010). For analyses related to RQ1-3, data was missing from 29 teachers, who were excluded from the analyses $(n=104)$. To address RQ4, the research team used Stata 16 to conduct several multiple regression analyses, where teacher composite scores measuring self-reported perceptions of the quality of feedback and support received from school administrators were modeled as the independent variables, and teachers' domain and subdimension scores were modeled as the dependent variables. For analyses related to RQ4, data was missing for 70 teachers, who were excluded from the analyses $(n=63)$. The results of these various analyses are outlined below.

Preliminary Associations between BCI Domains \& Subdimensions. Table 03 displays means, standard deviations, and correlations for the BCI domains and subdimensions. Teachers' scores for the Instructional and Interpersonal domains were moderately correlated to one another $(r=.563, p<.01)$, suggesting that these subdimensions provide related yet distinct information about educators' belonging-centered practices in the classroom. Scores for each of the Instructional subdimensions were moderate-to-strongly correlated with the broader Instructional domain (Safety to Be Wrong: $r=.768$, $p<.01$; Decentering Teacher Authority: $r=.745, p<.01$; Mathematics to Know Myself \& My World: $r=.574, p<.01 ;$ High Standards \& Rigorous Support: $r=.698, p<.01)$. Similar effects were found for the Interpersonal subdimensions and the broader Interpersonal domain (Social \& Emotional Bridging: $r=.740, p<.01$; Communal Orientation: $r=.622, p<$ .01 ; Empathetic Awareness \& Support: $r=.793, p<.01)$. Empathetic Awareness \& Support was moderate-to-strongly correlated with all other BCI subdimensions, excepting Mathematics to Know Myself \& My World $(r=.194, p<.05)$. 
Conversely, Mathematics to Know Myself \& My World was weakly or non-significantly related to the other BCI subdimensions, excepting Social \& Emotional Bridging $(r=.481, p<.01)$.

Table 03

Bivariate Correlations and Descriptive Statistics of BCI Domains \& Subdimensions

\begin{tabular}{|c|c|c|c|c|c|c|c|c|c|c|c|}
\hline & Means & SDs & 1 & 2 & 3 & 4 & 5 & 6 & 7 & 8 & 9 \\
\hline 1. Instructional Belonging & 2.68 & .40 & - & & & & & & & & \\
\hline 2. Interpersonal Belonging & 2.22 & .49 & $.563 * *$ & - & & & & & & & \\
\hline 3. Safety to Be Wrong & 2.71 & .53 & $.768 * *$ & $.421 * *$ & - & & & & & & \\
\hline 4. Decentering Authority & 2.81 & .63 & $.745^{* *}$ & $.426^{* *}$ & $.508 * *$ & - & & & & & \\
\hline 5. Mathematics to Know & 2.14 & .61 & $.574 * *$ & $.377 * *$ & $.178^{*}$ & $.180^{*}$ & - & & & & \\
\hline 6. High Standards/Rigorous Support & 3.06 & .52 & $.698 * *$ & $.334 * *$ & $.501 * *$ & $.336^{* *}$ & $.187 *$ & - & & & \\
\hline 7. Social \& Emotional Bridging & 2.38 & .81 & $.373 * *$ & $.740 * *$ & $.228 * *$ & .156 & $.481 * *$ & .155 & - & & \\
\hline 8. Communal Orientation & 1.87 & .62 & $.290 * *$ & $.622 * *$ & $.193 *$ & $.355^{* *}$ & .072 & $.173 *$ & .065 & - & \\
\hline 9. Empathetic Awareness/Support & 2.42 & .60 & $.566^{* *}$ & $.793 * *$ & $.515^{* *}$ & $.458 * *$ & $.194 *$ & $.424 * *$ & $.383 * *$ & $.396 * *$ & - \\
\hline
\end{tabular}

$* p<.05 . * * . p<.01$.

BCI Effects for Student Engagement. The researchers hypothesized teachers' domain and subdimension scores would positively predict student engagement as measured by external classroom observation (i.e., CLASS-S Student Engagement) scores. At the domain level, both teachers' Interpersonal and Instructional supports for belonging predicted student engagement ( $b=.24, p=.03$, and $b=.27, p<.01$, respectively). This model explained $28 \%$ of the variance in teachers' CLASS-S Student Engagement scores $\left(R_{\text {adj }}^{2}=.28\right)$. In a model with all three Interpersonal subdimensions as independent variables, two subdimensions, Social \& Emotional Bridging and Communal Orientation, collectively predicted student engagement ( $b=.20, p=.04$, and $b=.36, p<.01$, respectively), accounting for $24 \%$ of the variance between CLASS-S Student Engagement scores $\left(R_{\text {adj }}^{2}=.24\right)$. Finally, in a model with all four Instructional subdimensions as independent variables, one subdimension, Decentering Teacher Authority, explained $34 \%$ of the variance in CLASS-S Student Engagement scores $\left(b=.52, p<.01 ; R_{\text {adj }}^{2}=.34\right)$.

The researchers also hypothesized BCI domains and subdimensions would positively predict student engagement as measured by student self-report survey (i.e., Captivate) scores. At the domain level, the Interpersonal domain predicted student engagement $(b=.33, p<.01)$, while the Instructional domain did not $(b=-.06, p=.62)$. This model explained $6 \%$ of the variance in students' Captivate scores $\left(R_{\text {adj }}^{2}=.06\right)$. In a model with all three Interpersonal subdimensions as independent variables, two subdimensions, Social \& Emotional Bridging and Communal Orientation, predicted student engagement ( $b=.40, p<.01$, and $b=.31, p<.01$, respectively), explaining $15 \%$ of the variance between Captivate scores $\left(R^{2}{ }_{\text {adj }}=.15\right)$. There were no Instructional subdimensions that significantly predicted students' Captivate scores.

BCI Effects for Student Mathematical Agency. The researchers hypothesized BCI domains and subdimensions would positively predict students' mathematical agency as measured by student self-report survey (i.e., Confer) scores. At the domain level, only Interpersonal supports for belonging predicted students' mathematical agency and only marginally so $\left(b=.21, p=.08 ; R^{2}{ }_{\text {adj }}=.10\right)$ However, in a model with all three Interpersonal subdimensions as independent variables, two subdimensions, Social \& Emotional Bridging and Communal Orientation, explained $12 \%$ of the variance in Confer scores $\left(b=.29, p<.01\right.$, and $b=.25, p=.03$, respectively; $\left.R_{\text {adj }}^{2}=.12\right)$. Finally, in a model with all four Instructional 
subdimensions as independent variables, two subdimensions, Decentering Teacher Authority and Mathematics to Know Myself \& My World, collectively predicted students' mathematical agency $(b=.34, p<.01$, and $b=.18, p=.07$, respectively), explaining $13 \%$ of the variance between students' Confer scores $\left(R_{\text {adj }}^{2}=.13\right)$.

BCI Effects for Student Year-end Mathematics Achievement. The researchers hypothesized that BCI domains and subdimensions would positively predict students' year-end mathematics achievement as measured by the Balanced Assessment in Mathematics (BAM). In the analyses, the majority of the domains and subdimensions did not predict students' year-end mathematics achievement. However, one Instructional subdimension, Decentering Teacher Authority, did predict students' year-end mathematics achievement in a model with all four subdimensions as independent variables $(b=.29, p<.01)$, explaining $29 \%$ of the variance between students' BAM scores $\left(R_{\text {adj }}^{2}=.29\right)$.

Administrator Support \& Feedback for Teachers Effects for BCI. Finally, the researchers hypothesized that teachers' self-reported perceptions of the quality of feedback and support they received from school administrators would positively predict their BCI domain and subdimension scores. However, no teacher perceptions of the feedback and support they received from school administrators predicted their domain or subdimension scores.

\section{Discussion}

These results have several implications. First, the randomization of students into classrooms increases the confidence in and robustness of these findings. This randomization feature worked towards producing statistical equivalence between the observed classrooms, helping negate statistically significant differences between measured, unmeasured, and unimagined classroom- and student-level variables in this study (Remler \& Van Ryzin, 2010). Randomization thus prevents potential confounding classroom characteristics (e.g., lower-quality teachers, higher-performing students) from unduly influencing study outcomes. Overall, the randomization feature elevated the validity of the results in a way that could not be accomplished with traditional predictive analyses of classroom-based instruction on student outcomes. This study's findings, in conjunction with the study's random classroom assignment, collectively reinforce the relationship between belonging-centered teaching practices and positive student outcomes.

The implications and explanations of the results from the individual analyses described above will be discussed below.

Implications of Correlational Analysis. In the correlational analysis, Empathetic Awareness \& Support (EA\&S) was moderate-to-strongly correlated with all domains and subdimensions except Mathematics to Know Myself \& My World. This might indicate that characteristics of $E A \& S$ are related to the enactment of other subdimensions and domains. For example, teachers who demonstrate empathy might better understand students' emotions, difficulties, and circumstances, thus allowing them to acknowledge and attend to students' various needs. This attention to context might support teachers' ability to recognize when other belonging-centered practices are appropriate or needed.

Conversely, Mathematics to Know Myself \& My World (M2K) was not moderately or strongly correlated to many other subdimensions. This could indicate that $M 2 K$ is a belonging-centered practice that is conceptually unique from the other subdimensions. For example, while $M 2 K$ incorporates distinct instructional procedures similar to the other instructional subdimensions, these practices focus less on the techniques used when delivering mathematics instruction and more on how teachers can structure the content to be belonging-friendly. Despite its conceptual distinction, $M 2 K$ was still found to correlate statistically significantly with the Interpersonal and Instructional domains, suggesting that it is a relevant component of belonging-centered practice.

Implications of Regression Analyses Predicting Student Outcomes.

Student Engagement. In the domain-level student engagement analysis, both the Interpersonal and Instructional domains significantly predicted student engagement as measured by external CLASS-S observation scores. The results of this analysis indicate that teachers' support for students' Interpersonal and Instructional belonging needs within the classroom might better facilitate students' attentiveness and participation in mathematics.

In the subdimension-level analyses, most Instructional subdimensions did not significantly relate to student engagement. However, Decentering Teacher Authority (DTA) predicting CLASS-S Student Engagement scores indicates that instructional practices that promote student autonomy and agency are meaningfully related to one form of student engagement. DTA specifically may relate to engagement, because its action indicators (e.g., Engaging Students' Expertise, Opportunities for Agency, Inviting Feedback on Math Instruction) invite greater student participation and bidirectional interactions between the teacher and students. This indicates that teachers can leverage DTA action indicators to support both greater student belonging and engagement. Second, the Interpersonal domain and two of its subdimensions (Social \& Emotional Bridging, Communal Orientation) predicting two different measures of student engagement (i.e., external classroom observations and student self-report measures) reinforces the robustness of the relationship between Interpersonal belonging supports and student engagement. 
Student Math Agency. Few studies have examined the link between belonging-centered teaching practices and student agency. Thus, the association between four subdimensions of Interpersonal and Instructional belonging supports (Social \& Emotional Bridging, Communal Orientation, Decentering Teacher Authority, and Mathematics to Know Myself \& My World ) and students' mathematical agency in the current study adds substantively to the body of belonging literature. Student agency is a particularly essential outcome for students of color, who frequently attend mathematics classrooms that neglect their cultural assets (Martin, 2008, 2013, 2015) and restrict opportunities for them to share their ideas, perspectives, and experiences (Gutierrez, 2012). This can have adverse implications for racially disenfranchised students' learning equity (i.e., their access to rigorous, identify-affirming, and empowering instruction; Gutiérrez, 2012), since their voices and identities have historically been marginalized. Thus, the results from this analysis might indicate that belonging-centered teaching practices aligned with the four subdimensions outlined above can support learning equity for students of color by honoring their ideas, input, and experiences (i.e., their agency). These four subdimensions accomplish this by promoting student autonomy (i.e., Decentering Teacher Authority), leveraging students' cultural funds of knowledge (i.e., Mathematics to Know Myself \& My World), centering students' personal experiences (i.e., Social \& Emotional Bridging), and encouraging communal work and interactions (i.e., Communal Orientation) in the mathematics classroom.

Standardized Mathematics Achievement. Although most subdimensions did not predict student achievement, Decentering Teacher Authority (DTA) was significantly associated with students' standardized test scores. There are several potential explanations for DTA being an exception in these analyses. First, teachers that enact DTA encourage students to engage in conceptual mathematical thinking by 1) explaining and justifying their reasoning, and 2) encouraging them to explore concepts using their own language and processes. Because the standardized assessment used in these analyses (i.e., the BAM) assesses several categories of mathematical thinking (i.e., not specific mathematics content), students that have participated in classrooms that encourage higher-order thinking would likely achieve higher scores on the assessment. Second, DTA is associated with higher levels of student engagement and agency (see above), both of which are also related to higher achievement. This finding highlights how belonging-centered teaching practices that promote student autonomy and agency may support students' mathematics achievement.

Implications of Regression Analyses Predicting Teachers' Enactment of BCI. Teachers' perceptions of the quality of feedback and support they received from school administrators were not associated with BCI subdimensions and domains. There are several possible explanations for this finding. First, only a small subsample of teachers $(n=63)$ from the broader study sample $(N=133)$ completed the teacher surveys, which may have resulted in insufficient statistical power to predict any of these relationships. Second, implicit teacher beliefs about teaching, mathematics, and their students were not evaluated in the teacher survey. These might be important control variables in an analysis of how the quality of support and feedback they receive from their administration relates to their enactment of BCI, because these implicit beliefs can shape how teachers respond to and integrate the feedback they receive from their administration. Future analyses will continue to explore how school administrations nurture or thwart teachers' enactment of BCI to better support teachers in developing more belonging-centered practices.

\section{Use Case 02: Using the BCI Protocol to Document the Dynamic Interplay of Interpersonal and Instructional Subdimensions through State-Space Grids}

The variability in and across the BCI subdimensions allows room to examine multiple facets of teaching. One particular facet that the researchers examined through the BCI subdimensions was warm demander pedagogy, whereby teachers provide a nurturing and rigorous instructional environment to facilitate academic achievement for students of color (Ware, 2006). Warm demander pedagogy has been theorized to be particularly empowering for students of color (Ford \& Sassi, 2014; Ware, 2006), because the rigorous support and nurturing interpersonal interactions that they receive from warm demanding teachers can address the low expectations (Nasir \& Shah, 2011; Okeke et al., 2009; Pena-Shaff et al., 2019) and insufficient resources (Darling-Hammond, 2013; Frankenberg, 2013) that they often encounter in mathematics classrooms. Utilizing State-space grids (SSG) to graphically evaluate teachers' enactment of warm demander pedagogy, the research team examined the interactions between teachers' engagement with two distinct BCI subdimensions that align with the pedagogy's objectives: High Standards \& Rigorous Support (HS\&RS; i.e., instructional belonging supports) and Empathetic Awareness \& Support (EA\&S; i.e., interpersonal belonging supports). This analysis was guided by the following research question: What are the similarities and differences between patterns of teachers' enactment of High Standards \& Rigorous Support and Empathetic Awareness \& Support (i.e., warm demander pedagogy) as represented by SSGs?

\section{Method}

From the study's sample of 399 middle school mathematics classroom video recordings, three with high scores (i.e., 5 or above) for both $H S \& R S$ and $E A \& S$ were selected for further analysis. These videos included diverse enactments of these subdimensions, allowing for a dynamic range of classroom interactions to evaluate. The research team developed a 
four-point scale to evaluate the quality of each subdimension that ranged from 0 (i.e., the subdimension was absent) to 3 (i.e., the subdimension was present at a high level). Teacher-student interactions were categorized as distinct events, where any change in the quality of either $H S \& R S$ or $E A \& S$ in the teacher's instruction was defined as a new and unique event. Events where at least one subdimension received a score of 3 were categorized as "high-quality" events. Events where at least one subdimension received a score of 2 but neither subdimension received a score greater than 2 were categorized as "moderate-quality" events. Events where at least one subdimension received a score of 1 but neither subdimension received a score greater than 1 were categorized as "low-quality" events. While low-quality events were characterized just by teachers' use of encouraging and supportive language, moderate- and high-quality events also included actions that addressed students' academic and socioemotional needs. Three members of the research team used this protocol to score the quality of $H S \& R S$ and $E A \& S$ interactions for the first 30 minutes of each of the three classroom videos, after which they met to develop a consensus on the scores.

\section{Results}

Figure 03 below displays the results of this analysis and shows the distributions of $H S \& R S$ and $E A \& S$ interactions for each of the three teachers. Quality scores of $H S \& R S$ and $E A \& S$ are represented along the y-axis and x-axis, respectively. Each dot on the grids represents a single event, all of which were plotted according to their assigned quality scores for $H S \& R S$ and $E A \& S$. Dots were then connected based on the chronological order of events. The size of each dot is proportional to the event's duration, where larger dots indicate longer events, and smaller dots indicate shorter events.

\section{Figure 03}

State-Space Grids of Three Middle School Mathematics Teachers

Teacher 01

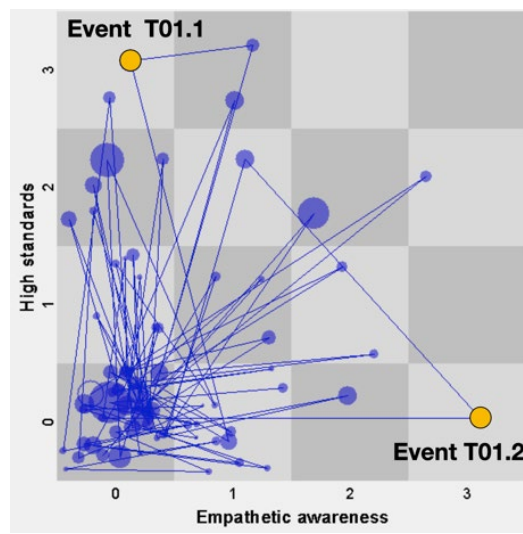

Teacher 02

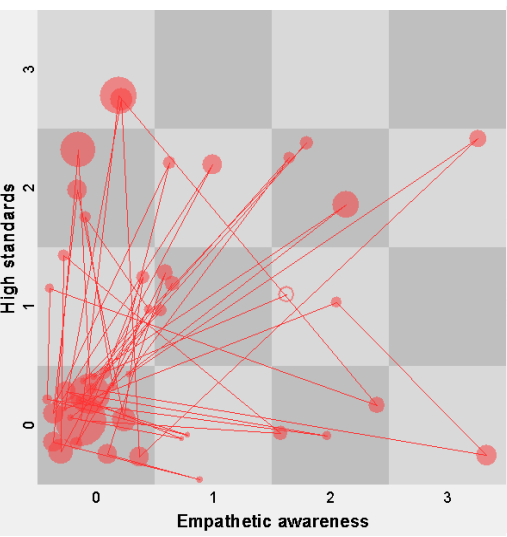

Teacher 03

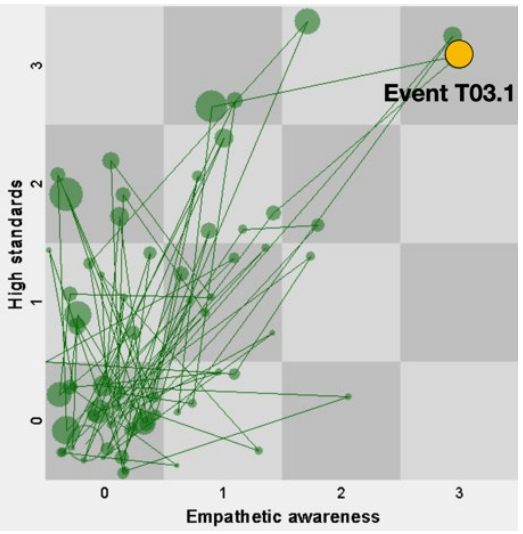

In this analysis, the research team discovered several differences and similarities in teachers' enactment of warm demander pedagogy, as visualized by the SSGs. This section begins by describing the distinctions in the distributions (i.e., patterns of where dots are scattered and clustered in the SSG) of classroom events for the three teachers' engagement with $H S \& R S$ and $E A \& S$, including the way teachers' actions reflected either singular enactments (i.e., demonstrating only one subdimension) or dual enactments (i.e., demonstrating both subdimensions simultaneously). Next, this section outlines how the proportion and duration of classroom events reflecting moderate- or high-quality warm demander pedagogy overlapped for the three teachers, indicating similarities across their teaching patterns.

Alternating Singular Enactments of BCI Subdimensions. The SSGs for Teacher 01 and Teacher 02 in Figure 03 revealed several overlaps in the distribution of the teachers' $H S \& R S$ and $E A \& S$ practices. Both teachers spent more time engaging singular enactments than dual ones throughout the instructional segment $(92.4 \%$ and $84.0 \%$ more time for Teacher 01 and Teacher 02 , respectively), which indicates that they more frequently addressed the two subdimensions of student belonging individually versus simultaneously. However, both teachers frequently alternated between singular $H S \& R S$ and $E A \& S$ enactments, which demonstrates that they provided both instructional and interpersonal supports for belonging by fluidly and contextually moving between both subdimensions across the broader classroom period. Further, many of these singular enactments received quality scores of 2 or $3(66.8 \%$ and $87.8 \%$ for Teacher 01 and Teacher 02 , respectively), which reveals that these teacher actions were moderately or highly meaningful for supporting both domains of student belonging.

To highlight how the two teachers engaged both subdimensions of student belonging across the broader instructional segment, we analyzed two of Teacher 01 's singular enactments: one high-quality enactment of $H S \& R S$ and one of $E A \& S$. 
In the first example (Score: 3 [HS\&RS], 0 [EA\&S]; Duration: 47s; see Event T01.1 in Figure 03), Teacher 01 communicated high standards and shared problem-solving strategies, which supported students' instructional belonging by pushing them to achieve academically. At the beginning of this event, Teacher 01 pressed a student to clarify their unit of measurement (" 1 and $3 / 4$ what? 1 and $3 / 4$ what?"). After the student responded ("Inches"), Teacher 01 emphasized the importance of labeling units of measurement ("Please be very, very specific. The measures do matter here. They do matter... No ifs and buts about it. You must label your work."). However, beyond just highlighting the importance of labeling units of measurement, Teacher 01 also explained why doing so mattered for the students' achievement ("We are looking at part two of the [state standardized] test, and they expect us to show every part of the solution"). Further, Teacher 01 connected labeling units of measurement to the broader problem-solving process and outlined her expectations for students when working through problems on the standardized test ("When I say process, when I say this word, I want you to show me how you solve the problem step by step... And you must label everything if you want that full credit on part two"). These actions collectively demonstrated the blend of communicating high standards with sharing problem-solving strategies that characterizes $H S \& R S$.

In addition to meeting students' instructional belonging needs through singular enactments of $H S \& R S$, Teacher 01 also supported students' interpersonal belonging through high-level singular enactments of $E A \& S$. For example, during one such event (Score: 0 [HS\&RS], 3 [EA\&S]; Duration: 46s; see Event T01.2 in Figure 03), Teacher 01 prioritized a student's wellbeing by stopping instruction when she recognized that student's physical distress ("What's the matter, sweetheart? You're not feeling well? You sick? Your head hurts?"). However, beyond merely acknowledging the student's discomfort, Teacher 01 also provided the student with emotional support. After creating space for a private discussion, Teacher 01 took the student's temperature, expressed sympathy for her discomfort, and inquired if the student felt well enough to continue with the lesson. Overall, this event highlighted the sensitivity to and support of students' physical and emotional health that characterizes $E A \& S$.

Taken together, these two singular enactments of $H S \& R S$ and $E A \& S$ demonstrated one pathway through which the teachers engaged both instructional and interpersonal subdimensions of student belonging across a broader class period. Although Teacher 01 and Teacher 02 more often enacted $H S \& R S$ and $E A \& S$ individually, they both fluidly alternated between the two subdimensions based upon the classroom context. In certain instances, the teachers supported students to achieve the high standards they communicated, while in others, they attended to students' socioemotional needs at a high level. Collectively, these practices ensured that students not only felt empowered to thrive academically (i.e., instructional belonging), but also felt comfortable and validated in the classroom space (i.e., interpersonal belonging). In other words, these teachers' instructional and interpersonal supports for belonging built upon each other across the broader classroom period to meet students' multidimensional belonging needs.

Blended Dual Enactments of BCI Subdimensions. While alternating singular enactments represented one option for meeting students' diverse belongingness needs, another pathway existed through dual enactments, which frequently materialized in Teacher 03's classroom. Teacher 03 spent more time leveraging dual enactments than singular ones $(7.5 \%$ more time), many of which were performed at a moderate or high level (i.e., 38.4\% of dual enactments received a score of $[2,2]$ or higher). The consistent frequency and high quality of Teacher 03's dual enactments illustrated how she was able to support students' interpersonal and instructional belongingness needs simultaneously.

To highlight how Teacher 03 supported students' interpersonal and instructional belonging at the same time, we analyzed one of her high-quality dual enactments. This dual enactment (Score: 3 [HS\&RS], 3 [EA\&S]; Duration: 83s; see Event T03.1 in Figure 03) was characterized by Teacher 03 supporting a student in meeting her high mathematical standards while concurrently attending to the student's academic and emotional needs. During the event, Teacher 03 noticed and acknowledged the student's confusion while he was completing his independent practice ("You have a question, baby doll? You're having trouble?"), and then reviewed his work to identify the specific difficulty ("I think you're missing something"). To address his uncertainty, Teacher 03 reassured and expressed a willingness to partner with the student to address the underlying causes of the difficulty ("If you can't get it, I will absolutely help you, because this is all kind of new"). However, this interaction moved beyond merely discussing the student's difficulties. Teacher 03 also highlighted the student's effort ("I can see you set up good"; "You did this already. So far, so good") and communicated high expectations by pushing the student to stretch and refine his conceptual and procedural thinking ("What does this have to do with this? What relationship do they have? Right, now tell me what an opposite means? Right, so going back to this, what can we do?").

Overall, this event demonstrated how Teacher 03 leveraged two subdimensions of BCI concurrently to meet students' multifaceted belongingness needs. Teacher 03's sensitivity to the student's unique difficulties, affirmations of his effort, and expression of partnership supported his interpersonal belonging by deepening social ties and his level of comfort. Further, her simultaneous provision of high standards and extensive scaffolding also supported the student's instructional belonging by easing the learning process and bolstering his mathematical self-efficacy. 
Similarities Between Teachers. Despite these distinctions between the three teachers, there were several notable similarities as well. First, all three teachers spent a greater proportion of their classroom time engaging in moderate- to highquality events (Teacher 01 , Teacher 02 , and Teacher 03 spent $73.4 \%, 84.2 \%$, and $67.5 \%$ of classroom time engaging in such events, respectively) compared to low-quality events. In these moderate- and high-quality events, the teachers went beyond merely using encouraging and supportive language (i.e., low-quality events), instead also engaging in identifiable actions that addressed students' academic and socioemotional needs. While this similarity was likely a function of the research team having selected classrooms with high $H S \& R S$ and $E A \& S$ Quality scores for the analysis, it indicates that that these teachers' warm demander pedagogies were characterized by both language and action that supported students' socioemotional and academic growth within the mathematics classroom.

Second, the average duration of the three teachers' moderate- and high-quality events was greater than that of their low-quality events. Teacher 01, Teacher 02 , and Teacher 03 spent an average of 38.6 seconds, 53.9 seconds, and 40.2 seconds engaging in moderate- or high-quality events, respectively, versus 9.7 seconds, 14.0 seconds, and 15.9 seconds for low-quality events. The distinctions between the average durations of moderate/high-quality events and low-quality events indicates that active and targeted support (i.e., moderate- and high-quality events) required more time from the teachers than passive and broad support. While this finding might seem intuitive, it highlights that the three teachers' warm demander pedagogies were characterized by the willingness to invest extended time and attention to support students' academic and socioemotional needs.

\section{Discussion}

Taken together, the similarities and differences between the three SSGs presented here highlight the dynamism of a warm demander pedagogy. The three teachers' high-quality and consistent engagement with both $H S \& R S$ and $E A \& S$ indicates that they rigorously pushed and supported students within a nurturing framework of interpersonal interaction, which highlights the presence of a warm demander pedagogy in each of the classrooms. However, the distinctions between teaching patterns, represented through SSGs (e.g., singular versus dual enactments) illustrates that a warm demander pedagogy can materialize differently across classrooms. While some teachers might contextually and fluidly alternate between communicating high expectations, pushing students to meet them, and supporting students' emotional needs across an instructional period, other teachers blend these actions together within the context of individual interactions with students. These findings add to the warm demander pedagogy literature by not only suggesting that warm demander pedagogy can be enacted differently across unique classroom contexts, but also by illustrating the distinct ways that teachers may enact such a pedagogy in their classrooms.

Overall, this analysis highlights how SSGs can be paired with the BCI protocol to better understand the complexities and nuances of belonging-centered teaching. While the regression models employed in Use Case 01 are important for understanding how BCI relates to student outcomes, they do not capture the dynamism of BCI nor the intricacies of BCI practices. However, SSGs allowed for this dynamism and nuance to be graphically visualized, where entire classroom episodes could be broken down into a series of individual interactions that could be examined both in isolation and in relation to one another. Doing so allowed for an understanding of how high-quality BCI practices can vary across distinct classroom contexts (e.g., alternating singular enactments versus blended dual enactments) while still supporting students' multidimensional belonging needs. Thus, SSGs provide educational stakeholders with a novel tool to visually identify the specific teaching practices and processes - as well as the relationships between them - that nurture student belonging. Researchers and practitioners will benefit from the deeper understandings of the complexities and dynamism of belongingcentered teaching practices that SSGs yield, and they will also have access to contextualized examples of high-quality BCI that can be integrated into real classrooms.

While this SSG analysis provided several insights about warm demander pedagogy specifically and the BCI protocol more broadly, it also highlights a need for further research. First, it was unclear whether distinctions between teachers' styles of warm demander enactment (i.e., singular versus dual enactment) had any implications for students. The research team plans to address this by examining whether teachers' varied enactments of warm demander pedagogy relate to specific student outcomes, including engagement, achievement, and sense of belonging. Further, the research team did not explore whether certain teacher-level factors (e.g., years of teaching experience, support from administrators) relate to teachers' enactment of warm demander pedagogy. Future analyses can consider whether these teacher-level factors are associated with singular or dual enactments of the $H S \& R S$ or $E A \& S$ subdimensions of BCI. 


\section{Belonging-Centered Instruction (BCI) Observational Protocol}




\section{Protocol Overview and Key Terms:}

This observational protocol is designed to help the user assess a teacher's enactment of Belonging-Centered Instruction (BCI). BCI is a pedagogical framework that defines and assesses how teachers create opportunities for active inclusion, achievement, identification, and empowerment in the classroom through interpersonal and instructional techniques. This observational protocol helps the observer evaluate the degree to which a teacher's actions facilitate students' sense of belonging within mathematics classrooms. Note: The effective and accurate use of this protocol requires extensive practice and training from the authors. Use of this protocol before training will rely in unreliable scores and outcomes.

This protocol and BCI more broadly encompass two domains of belonging support: 1) Interpersonal Belonging Supports and 2) Instructional Belonging Supports. Interpersonal Belonging Supports include educator practices that actively facilitate social ties, social comfort, and interconnectedness between the members of a classroom. As a result of these practices, students will ideally feel respected, seen, and valued as a member of the classroom community. Instructional Belonging Supports include educator practices that use instruction to help students make meaningful connections between the content, the learning process, their identities, and broader society. As a result of these practices, students will ideally see themselves as capable doers of mathematics and see the importance of math for their lives and the world around them.

Each domain comprises several unique subdimensions, each of which represents a set of related teacher actions that support either interpersonal or instructional opportunities for belonging. For each subdimension, a description has been provided that specifically outlines the broader pattern of teacher actions that the subdimension encompasses. For a comprehensive list of the subdimensions and their descriptions, see Table 01.

\section{Table 01: BCI Subdimensions \& Descriptions}

\begin{tabular}{|c|c|}
\hline Interpersonal Belonging Supports & istructional Belonging Supports \\
\hline $\begin{array}{l}\text { Social \& Emotional Bridging } \\
\text { Teacher creates a positive classroom environment that } \\
\text { deconstructs social boundaries between teacher and students. } \\
\text { Teacher shares their humanity (e.g., opinions, likes, stories, } \\
\text { personal details) and seeks to relate to students in ways that } \\
\text { recognize students' humanity (i.e., seeing them as whole people, } \\
\text { not just as students). Teacher and students share emotions and } \\
\text { experiences that help each other become "seen" and "known." } \\
\text { Communal Orientation } \\
\text { Teacher promotes a "we're in this together" orientation with } \\
\text { their students. Teacher supports a climate of communalism over } \\
\text { individualism across the social dynamics within the classroom. } \\
\text { Teacher maintains a vested interest in the academic and social } \\
\text { growth of the group, and in relying on one another to achieve } \\
\text { that growth. } \\
\text { Empathetic Awareness \& Support } \\
\text { Teacher is conscious of individual students' strengths, emotions, } \\
\text { physical wellbeing, and resource needs. Teacher supports } \\
\text { individual students' emotional and psychological health, and } \\
\text { demonstrates that individual students matter in the life of the } \\
\text { classroom. Teacher emphasizes students' value regardless of } \\
\text { mathematical ability. }\end{array}$ & $\begin{array}{l}\text { Safety to Be Wrong } \\
\text { Teacher creates a space where students do not feel stigmatized } \\
\text { for wrongness or needing support. Teacher disarms and } \\
\text { normalizes wrongness. Students have the opportunity to break } \\
\text { the pattern of judging their mathematical belongingness simply } \\
\text { based on correctness. Mastery and effort are celebrated over } \\
\text { performance and talent. } \\
\text { De-centering Teacher Authority } \\
\text { Teacher indicates that students' mathematical methods/ideas } \\
\text { have real value (worth). Teacher positions students as } \\
\text { knowledgeable authorities in mathematics. Students have a } \\
\text { sense that their intellectual contributions matter in the life of this } \\
\text { classroom. } \\
\text { Mathematics to Know Myself \& My World } \\
\text { Teacher provides opportunities to use math to support student } \\
\text { agency, empower cultural identity, understand the world, and } \\
\text { critique their social world. } \\
\text { High Standards \& Rigorous Support } \\
\text { Teacher communicates high standards for students while also } \\
\text { providing support to help students achieve these standards. } \\
\text { Teacher implicitly or explicitly communicates that they expect } \\
\text { consistent mathematical effort and high-level performance. } \\
\text { However, beyond high standards and support, the teacher } \\
\text { cultivates the belief within students that they can fulfill the high } \\
\text { expectations by instilling confidence and/or sharing strategy. }\end{array}$ \\
\hline
\end{tabular}


Each subdimension has its own section of the protocol. These sections, called subdimension Scoring Sheets, include supplementary information and procedures that support the scoring of each subdimension.

The top half of page one of each subdimension's Scoring Sheets is called the Subdimension Details, and it includes the subdimension name, its description, and its corresponding action indicators, which are the teacher actions that work towards fulfilling the subdimension description. See Figure 01 below for an example of how this section is arranged.

\section{Figure 01: Sample Subdimension Details}

\begin{tabular}{|c|c|c|}
\hline \multirow[t]{2}{*}{$\begin{array}{l}\text { Subdimension Name- } \\
\text { Subdimension } \\
\text { Description }\end{array}$} & \multicolumn{2}{|r|}{$\begin{array}{l}\text { SAFETY TO BE WRONG } \\
\text { Teacher creates a space where students do not feel stigmatized for wrongness or needing support. Teacher disarms and } \\
\text { normalizes wrongness. Students have the opportunity to break the pattern of judging their mathematical belongingness simply } \\
\text { based on correctness. Mastery and effort are celebrated over performance and talent. }\end{array}$} \\
\hline & \multirow{5}{*}{ 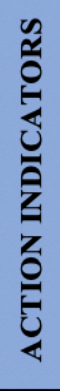 } & Normalizing Difficulties: Teacher shows that wrongness is a normal and expected part of the learning process. \\
\hline \multirow{4}{*}{ Action Indicators } & & $\begin{array}{l}\text { Creating Space for Questions: Teacher seeks out questions from students, creating an environment that is a safe place } \\
\text { for uncertainty and learning. }\end{array}$ \\
\hline & & $\begin{array}{l}\text { Task-focused Encouragement: Teacher attempts to disarm self-doubt and encourages students to persist through } \\
\text { difficult mathematical tasks. }\end{array}$ \\
\hline & & $\begin{array}{l}\text { Celebrating Mastery-focused Behavior: Teacher celebrates students' effort or strategies as examples of how to } \\
\text { overcome difficulties on the road to mastery. }\end{array}$ \\
\hline & & $\begin{array}{l}\text { Modeling Mistakes for Learning: Teacher models their own or students' mistakes as potential teaching moments for } \\
\text { students. Teacher acknowledges mistakes/errors and uses them to create opportunities for learning. }\end{array}$ \\
\hline
\end{tabular}

The bottom half of the first page of each subdimension's Scoring Sheet, called Scoring Details, includes procedures on scoring that subdimension. These will be discussed more extensively below in the "Procedures for Observing \& Scoring Classrooms Using the BCI Observational Protocol" section of this introduction (below).

Page two of each subdimension's Scoring Sheets (called Observation Notes) provides space for the observer to 1) record descriptive information about the classroom that they are observing (e.g., date, observer's name, classroom, teacher, grade, topic), and 2) take notes on what they observe in the classroom. Please refer to the "The Observational Phase" section of this introduction for more detailed information about observational procedures and note-taking.

Page three or pages three and four of each subdimension's Scoring Sheets include the Action Indicator Table, which provides information about the subdimension's action indicators. The first column of this table includes each action indicator and the teacher behaviors that are associated with each action indicator (see Figure 02 below). Action indicators have two to three associated behaviors listed to assist observers in better identifying when teachers are enacting the action indicators in the classroom. They are also essential in helping observers evaluate how strongly and consistently the teacher performs a particular action during the observation (see more on this in the "Scoring" section of the introduction).

The first three columns of this table include examples of what each action indicator might look like in real classrooms. These examples will help the observer understand how the action indicator and its associated behaviors might materialize during an observation. They have also been categorized as Early, Developing, or Advanced, which allows observers to better distinguish between low-, mid-, and high-strength materializations of action indicators for scoring purposes (see more on this in the "Scoring Quality" section of the introduction). Please note that these examples are for guidance and are not fully exhaustive of what might happen in the classroom.

Figure 02: Portion of a Sample Action Indicator Table

\begin{tabular}{|c|c|c|c|c|}
\hline & Action Indicators & Early & Developing & Advanced \\
\hline $\begin{array}{l}\text { Action Indicator }- \\
\text { Behaviors } \\
\end{array}$ & $\begin{array}{l}\text { Normalizing Difficulties: } \\
-\quad \text { Shows errors are normal. } \\
-\quad \text { Attempts to remove negative } \\
\text { emotions associated w/ errors. } \\
\text { Shows wrongness is part of } \\
\text { learning. }\end{array}$ & $\begin{array}{l}\text { When a student provides an } \\
\text { incorrect answer, the teacher } \\
\text { responds, "That's okay!"” } \\
\text { before moving on to another } \\
\text { student. }\end{array}$ & $\begin{array}{l}\text { When a student provides an incorrect } \\
\text { answer, the teacher responds, "That's } \\
\text { fine! We all make mistakes sometimes. } \\
\text { Now, let's fix it!" }\end{array}$ & $\begin{array}{l}\text { When having students correct homework, the teacher says, } \\
\text { "If you made a mistake, don't stress it. It's the corrections } \\
\text { that are important. Corrections help you identify what you } \\
\text { did wrong and how you can do better next time." }\end{array}$ \\
\hline
\end{tabular}


The final page of each subdimension's Scoring Sheets includes the Equity Statement, which highlights how that particular subdimension promotes learning equity and/or humanizes mathematics. This reminds observers to keep equity and belonging as the guiding framework of their observations and scoring.

\section{Procedures for Observing and Scoring Classrooms Using the BCI Observational Protocol:}

Observing and scoring a classroom can be challenging, especially when doing so in real time. A classroom is a busy and dynamic environment, and it can be difficult to track the many and diverse interactions and choices that teachers make across a 45-minute observational cycle. Scoring a classroom based on that observation presents its own challenges, and it can be overwhelming trying to assign a quantitative score that acknowledges the potential conflicts and honors the various distinctions that materialize in the classroom. For these reasons, we conceptualize the observation-scoring process in two distinct but consecutive phases: 1) the Observational Phase, and 2) the Scoring Phase.

\section{The Observational Phase:}

Before beginning an observation, we strongly encourage observers to consult the "Quick Start Guide" section of this protocol and to study its contents. This resource provides a concise two-page summary of this entire introduction, focusing on the primary considerations of the observation-scoring process. Once an observation begins, the observer should be consumed with the contents and occurrences of the classroom. Referencing the materials in this protocol too frequently midobservation will distract the observer from the classroom, potentially leading to missed interactions.

During the Observational Phase, observers will pay attention to 1) what the teacher is doing inside the classroom and 2) the descriptions of the subdimensions, which refer to patterns of teacher actions that promote student belonging in relation to that subdimension. In the Observation Notes of each subdimension's Scoring Sheets, observers should record things the teacher does that seem related to belonging and/or that align with the subdimension descriptions. Observers without much experience with the BCI observational protocol should keep their notes general, focusing more on subdimension descriptions and less on specific action indicators during this Observational Phase.

As observers become more experienced and familiar with the BCI observational protocol, they may feel more comfortable taking notes on specific action indicators. They may also naturally start to consider how a teacher's actions will affect the ultimate score while observing. However, we still strongly recommend 1) focusing on what the teacher is doing broadly and not on the action indicators narrowly, and 2) keeping the Observational Phase as distinct as possible from the Scoring Phase, so that observers do not become distracted from what is happening in the classroom by focusing on details or scores during the observation.

\section{The Scoring Phase:}

After the 45-minute observation cycle is completed, observers will refer to their notes and recall their impressions to provide quantitative scores of the subdimensions of BCI. Observers will provide two scores for each subdimension: one for Quality, and one for Variation. Quality indicates the degree to which the teacher's actions fulfill the description of the subdimension throughout the observation cycle. Variation refers to the range and/or quantity of different action indicators that were present throughout the observation cycle.

For both scores, an important consideration is each subdimension's action indicators, which relate to how well a teacher fulfills the subdimension's description (Quality) and how many different behaviors the teacher utilizes within each subdimension (Variation). Once the observation is over, observers should deeply consider how the teacher actions they observed (and that are reflected in their notes) relate to the action indicators across the various subdimensions (i.e., Were these action indicators present? How strong were they?). To do so, observers should consult the information in each subdimension's Scoring Sheets (e.g., the definitions of the action indicators in the Subdimension Details; the detailed information in each subdimension's Action Indicator Table), as they will help guide the observer's evaluation of the action indicators. These evaluations should be noted and integrated into both the Quality and Variation scores. Further instructions on how to do so are outlined in the "Scoring Quality" and "Scoring Variation" sections below. 


\section{Scoring Quality:}

Quality indicates the degree to which an observed teacher's actions fulfill the subdimension description throughout the observational cycle. Each subdimension will receive a separate Quality score, which represents a distinct pattern of teacher actions. When evaluating a subdimension's Quality, an important consideration is that subdimension's action indicators, because these reflect identified teacher actions that meaningfully work towards fulfilling that subdimension. The presence and/or strength of the action indicators can guide the observer to an appropriate Quality score.

To determine whether or not a teacher's action qualifies as an action indicator, observers should refer to the appropriate Subdimension Details for the action indicator's definition and Action Indicators Table for examples of behaviors associated with that action indicator.

\section{Figure 03: Extended Portion of a Sample Action Indicator Table}

\begin{tabular}{|c|c|c|c|c|}
\hline & \multicolumn{4}{|c|}{ Early, Developing, \& Advanced descriptions } \\
\hline Action Indicators & & Early & Developing & Advanced \\
\hline & 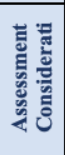 & $\begin{array}{l}\text { Teacher engages in one or } \\
\text { more behaviors in a brief } \\
\text { and/or superficial way. }\end{array}$ & $\begin{array}{l}\text { Teacher 1) engages in one behavior in a } \\
\text { meaningful way and the others not at all } \\
\text { or superficially, or 2) engages in two or } \\
\text { more behaviors in a way that falls } \\
\text { between superficial and meaningful. }\end{array}$ & $\begin{array}{l}\text { Teacher engages in two or more behaviors in an extended } \\
\text { and/or meaningful way. }\end{array}$ \\
\hline $\begin{array}{ll}\text { Normalizing Difficulties: } \\
-\quad \text { Shows errors are normal. } \\
-\quad \text { Attempts to remove negative } \\
& \text { emotions associated w/ errors. } \\
- & \text { Shows wrongness is part of } \\
& \text { learning. }\end{array}$ & & $\begin{array}{l}\text { When a student provides an } \\
\text { incorrect answer, the teacher } \\
\text { responds, "That's okay!" } \\
\text { before moving on to another } \\
\text { student. }\end{array}$ & $\begin{array}{l}\text { When a student provides an incorrect } \\
\text { answer, the teacher responds, "That's } \\
\text { fine! We all make mistakes sometimes. } \\
\text { Now, let's fix it!" }\end{array}$ & $\begin{array}{l}\text { When having students correct homework, the teacher says, } \\
\text { "If you made a mistake, don't stress it. It's the corrections } \\
\text { that are important. Corrections help you identify what you } \\
\text { did wrong and how you can do better next time." }\end{array}$ \\
\hline
\end{tabular}

If it is determined that an action indicator is present, the Action Indicator Table can also be helpful in determining the teachers' strength in performing that action indicator during the observation. At the top of this table, there are descriptions of what constitutes an Early, Developing, or Advanced display of the action indicator (see Figure 03). An Early action indicator would be one in which one or more of its associated behaviors (listed in the leftmost column; e.g., Shows errors are normal; Attempts to remove negative emotions associated with errors; Shows wrongness is part of learning) was enacted by the teacher in a brief and/or superficial way. An Advanced score for the action indicator is when the teacher enacts two or more of the associated behaviors for a longer period of time and/or in a meaningful way. Observers can also refer to that action indicator's examples to guide their understanding of strength, although it is important to note that these examples are not exhaustive.

It is critical for observers to remember that it is the overall subdimension being scored (i.e., to what degree its description was fulfilled), not the individual action indicators. Observers without much experience with the BCI observational protocol might be tempted to focus too much on the action indicators, using the descriptions of Early, Developing, and Advanced above to evaluate the strength of each indicator. This could lead them to base the subdimension's Quality score on these evaluations. However, the Action Indicator Tables are intended to function as guides to understand the many ways action indicators might show up in classrooms. They are helpful for developing a general impression of the action indicators' presence and strength and understanding how they might work toward fulfilling the subdimension description.

Similarly, the absence or presence of the action indicators themselves may not necessarily relate directly to a subdimension's description. There may be circumstances where a subdimension's description is strongly present in a teacher's actions without the presence of many action indicators (see the Are the action indicators exclusive? part of the FAQ section below), and there may likewise be circumstances where present action indicators do not work towards building the subdimension description in any meaningful way.

It is thus better to think of the action indicators as pointing towards the subdimension description (i.e., not being the same as it), and observers should focus more on the subdimension description when scoring Quality and less on the individual action indicators. While the presence, strength, and consistency of a subdimension's action indicators are important considerations of that subdimension's Quality, this broader view of the subdimension description will allow for more consistent and accurate Quality scores for that subdimension. In other words, when scoring Quality, observers should ask 
themselves, "To what degree did the teacher's actions (especially the action indicators) fulfill this subdimension's description?"

Once an observer has developed an answer to or assessment of this question, they should refer to the Quality portion of the Scoring Details (see Figure 04) for that subdimension. This section explicitly outlines what constitutes an Early, Developing, and Advanced Quality score.

Figure 04: Quality Portion of Sample Scoring Details

\begin{tabular}{|c|c|c|c|}
\hline & $\begin{array}{l}\text { Early } \\
(1,2)\end{array}$ & $\begin{array}{c}\text { Developing } \\
(3,4)\end{array}$ & $\begin{array}{c}\text { Advanced } \\
\qquad(5,6)\end{array}$ \\
\hline Quality & $\begin{array}{l}\text { The quality of teacher actions } \\
\text { consistently reflects Safety to Be } \\
\text { Wrong in a shallow, partial, } \\
\text { light, or inauthentic way }\end{array}$ & $\begin{array}{l}\text { The quality of teacher actions is } \\
\text { mixed/inconsistent or reflects } \\
\text { Safety to Be Wrong in a way that } \\
\text { falls between superficial and } \\
\text { meaningful }\end{array}$ & $\begin{array}{c}\text { The quality of teacher actions } \\
\text { consistently reflects Safety to Be } \\
\text { Wrong in a rich, significant, or } \\
\text { enduring way }\end{array}$ \\
\hline Score: & & & \\
\hline
\end{tabular}

In determining which of these scores best fits the teacher that was observed, it is important to be mindful of one additional consideration: Quality refers to consistency of Quality. Thus, teachers whose actions consistently align with the subdimension description in "superficial," "partial," or "inauthentic" ways are more likely to receive an Early Quality score. Teachers whose actions consistently align with the subdimension description in "rich," "significant," or "meaningful" ways are more likely to receive an Advanced Quality score. Teachers whose actions were inconsistent (i.e., sometimes "superficial" and sometimes "meaningful") or consistently fall somewhere between "superficial" and "meaningful" (i.e., of moderate strength) are more likely to receive a Developing Quality score. However, consistently does not mean exclusively; even teachers that receive an Advanced score are likely to engage in occasional superficial actions.

\section{Frequently Asked Questions about Scoring Quality:}

While the above section covered the core considerations related to scoring Quality, it is not exhaustive. Below are a number of frequently asked questions related to scoring Quality.

Are all action indicators weighted or interpreted equally?

This question is complex, but the simple answer is NO. There are many and varied ways teachers can enact action indicators across classrooms and instructional moments, and observers should expect that the meaningfulness and frequency of these action indicators will vary as well. In some classrooms, certain action indicators may meaningfully work towards fulfilling a subdimension's description due to the ways that the teacher enacts them. However, there may be other classrooms where these same action indicators may not meaningfully fulfill the subdimension description, because the teacher enacts them differently. Certain action indicators are also more likely to appear across some classrooms more than others, which can influence how observers conceive of and score Quality. Therefore, we strongly encourage observers to consider each action indicator - and teacher action more broadly — within its unique context, considering how consistently that action indicator is enacted in relation to the subdimension description for that specific classroom.

\section{Are subdimensions limited to just the available action indicators?}

While the action indicators are strong reflections of the overall subdimensions, they may not be fully exhaustive for every classroom. In certain circumstances, a teacher may perform an action that aligns well with the broader subdimension description but is not represented among the list of action indicators. In these rare circumstances, the observer should clearly delineate in the Observation Notes (the second page of that subdimension's Scoring Sheets) how that teacher action 1) differs from the other action indicators and 2) works toward fulfilling the subdimension description. After doing so, the observer may then integrate that teacher action into their considerations of how to score that subdimension's Quality. 
How do I decide between two numbers in the same score category (e.g., how do I decide between a 3 or a 4?)?

Each scoring category (i.e., Early, Developing, and Advanced) has two affiliated numerical scores (1 or 2 for Early, 3 or 4 for Developing, 5 or 6 for Advanced), After deciding which category is most appropriate for an observed classroom, the observer will decide which of the two scores fits that classroom best by using the system outlined in Table 02 below.

Table 02: Breakdown of Quality Score Categories

\begin{tabular}{|c|c|c|c|c|c|}
\hline \multicolumn{2}{|c|}{ Early } & \multicolumn{2}{|c|}{ Developing } & \multicolumn{2}{|c|}{ Advanced } \\
\hline 1 & 2 & 3 & 4 & 5 & 6 \\
\hline $\begin{array}{l}\text { Teacher actions } \\
\text { related to the } \\
\text { subdimension } \\
\text { description are } \\
\text { either absent or } \\
\text { only align with } \\
\text { the Early } \\
\text { category. }\end{array}$ & $\begin{array}{l}\text { Teacher actions } \\
\text { related to the } \\
\text { subdimension } \\
\text { description are } \\
\text { consistently } \\
\text { within the Early } \\
\text { category, but } \\
\text { some might fit } \\
\text { into the } \\
\text { Developing } \\
\text { description. }\end{array}$ & $\begin{array}{l}\text { Teacher actions } \\
\text { related to the } \\
\text { subdimension } \\
\text { description are } \\
\text { consistently } \\
\text { aligned with the } \\
\text { Developing } \\
\text { category, but some } \\
\text { might fit into the } \\
\text { Early description. }\end{array}$ & $\begin{array}{l}\text { Teacher actions } \\
\text { related to the } \\
\text { subdimension } \\
\text { description are } \\
\text { consistently aligned } \\
\text { with the Developing } \\
\text { category, but some } \\
\text { might fit into the } \\
\text { Advanced } \\
\text { description. }\end{array}$ & $\begin{array}{l}\text { Teacher actions } \\
\text { related to the } \\
\text { subdimension } \\
\text { description are } \\
\text { consistently aligned } \\
\text { with the Advanced } \\
\text { category, but some } \\
\text { might fit into the } \\
\text { Developing } \\
\text { description. }\end{array}$ & $\begin{array}{l}\text { Teacher actions } \\
\text { related to the } \\
\text { subdimension } \\
\text { description are } \\
\text { consistently or } \\
\text { nearly } \\
\text { exclusively } \\
\text { aligned with the } \\
\text { Advanced } \\
\text { category. }\end{array}$ \\
\hline
\end{tabular}

What do I do if I see no evidence for a particular subdimension in a classroom?

It is possible that, during the observation, none of the teacher's actions indicate that a particular subdimension is present. In these circumstances, that subdimension should receive a Quality score of 1 .

What do I do if I see only one teacher action related to the description, but that action is very meaningful?

In some cases, teachers may engage in a single rich or significant action that highly contributes to a subdimension. It may be tempting in these circumstances to assign an Advanced Quality score (i.e., a 5 or 6), because that action was meaningful. However, remember that Quality relates to consistency of Quality. A single teacher action, however meaningful, does not demonstrate consistency of Quality for that subdimension, unless that action endures for an extended period of time. Therefore, the teacher should not receive an Advanced Quality score, and will instead likely_although not necessarilyreceive a Developing score (i.e., a 3 or 4 ).

What do I do if I see teacher behaviors that work directly against the subdimension description?

In the "Scoring Quality" section of this Introduction above, we discussed only those teacher actions that work to fulfill a subdimension's description. When scoring a subdimension's Quality, these are the teacher actions that observers should focus on. In other words, observers should focus on the strengths that a teacher exhibits when evaluating the alignment between teacher actions and subdimension descriptions. However, there will occasionally be circumstances where a teacher engages in actions that directly contradict a particular subdimension (i.e., pose a threat to the belonging that that subdimension facilitates). In these circumstances, do NOT integrate these actions into your Quality score. We have created a separate Threats to Belonging subdimension (described below) that will evaluate teacher actions that undermine student belonging. A subdimension's Quality score should only account for those teacher actions that work towards fulfilling a subdimension's description.

Should I be focusing solely on teacher actions, or should I also consider students' responses to teacher actions?

Teacher actions will be met by varied responses from students. In some cases, teachers can effectively enact an element or subdimension of BCI, but students still appear disengaged or disinterested. It may be tempting to consider such student responses to teacher actions while scoring Quality, because they can be interpreted as illustrating the ineffectiveness of an element of BCI in that classroom. However, there are many unrelated factors — often beyond the teacher's control — that can influence student responses (e.g., time of the year, students' personal lives, broader school culture or sociopolitical context). Therefore, student responses are not always related to or indicative of the effectiveness of a teacher's actions, and they 
should NOT be considered when scoring a subdimension's Quality. Observers should instead focus only on teacher actions and the degree to which they fulfill a subdimension's description.

\section{Scoring Variation:}

Variation refers to the range and quantity of different action indicators that were present throughout the observation cycle. The Variation score recognizes the presence and frequency of action indicators, regardless of their Quality. We conceptualize Variation as being an important consideration within the BCI framework, because large and diverse classrooms require varied teacher actions to meet the unique needs of different students. Each subdimension will receive a distinct Variation score, as each subdimension's action indicators are unique.

Unlike scoring for Quality, which prioritizes the subdimension description, the Variation score focuses on quantity and diversity of the action indicators enacted during the observation period. To score a subdimension's Variation, observers should first identify the number of action indicators that occurred during the observation cycle for each subdimension and evaluate how frequently they were enacted. To determine whether or not a teacher's action qualifies as an action indicator, observers should refer to the action indicator definitions (in the Subdimension Details of each subdimension's Scoring Sheets) and their associated behaviors (listed in the Action Indicators Table in that subdimension's Scoring Sheets).

Once observers determine how many action indicators were present within a subdimension and how frequently each appeared, they should refer to the Variation portion of the Scoring Details (see Figure 05) on the first page of each subdimension's Scoring Sheets. This section explicitly outlines what constitutes an Early, Developing, and Advanced Variation score.

\section{Figure 05: Variation Portion of Sample Scoring Details}

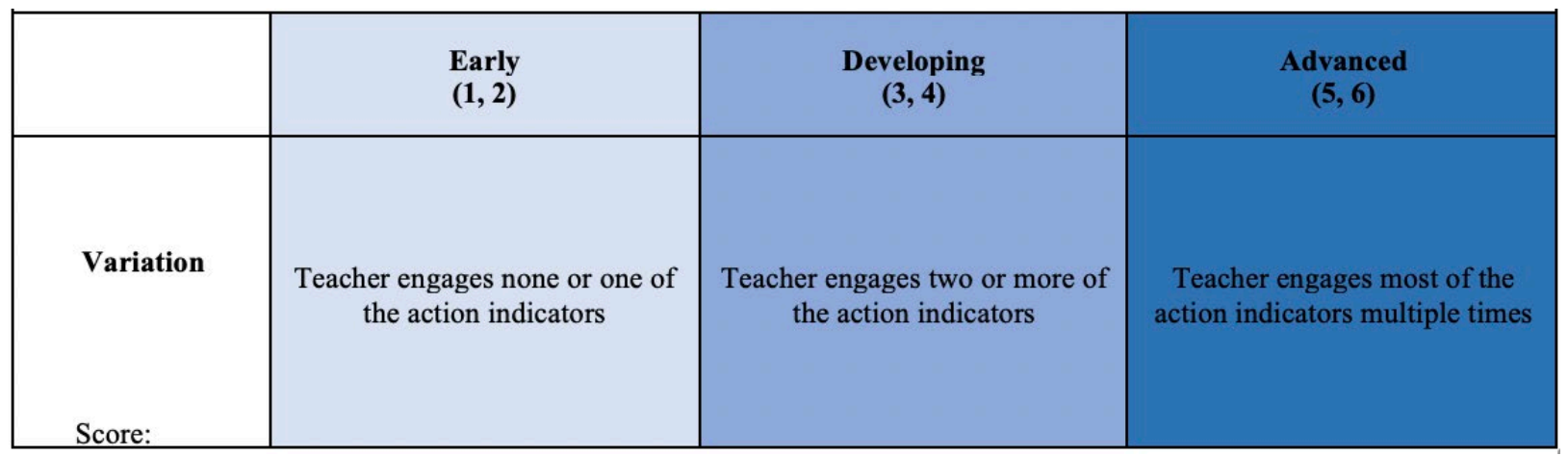

A teacher that enacts none or one of a subdimension's action indicators, regardless of frequency, would receive an Early Variation score for that subdimension. A teacher that enacts two or more of a subdimension's action indicators, with only one or some appearing multiple times, would receive a Developing Variation score for that subdimension. A teacher that enacts most (i.e., a majority) of a subdimension's action indicators multiple times would receive an Advanced Variation score for that subdimension. However, please note that a teacher does not have to perform all of the action indicators to receive an Advanced Variation score. It is unlikely and unrealistic to expect that a teacher will perform all of a subdimension's action indicators in one observation cycle, and their scores should not be reduced because of that.

\section{Frequently Asked Questions about Scoring Variation:}

Below are additional considerations and frequently asked questions related to scoring Variation.

\section{How is the Variation score distinct from the Quality score?}

Both the Quality and Variation scores are related to a subdimension's action indicators, but they are two distinct aspects of how that subdimension is enacted during the observation period. Variation describes the presence and frequency of action indicators regardless of their Quality. Thus, Variation and Quality scores can vary significantly within a single subdimension during one observation. For example, if a teacher engages in only one of a subdimension's action indicators 
but does so in a way that consistently and meaningfully fulfills the subdimension description, that subdimension could receive an Advanced Quality score but an Early Variation score. On the other hand, if a teacher enacts most of the subdimension's action indicators multiple times, but does so in ways that are consistently brief and superficial (i.e., do not fulfill the subdimension description in a meaningful way), they could receive an Early Quality score but an Advanced Variation score. While a subdimension's Quality and Variation scores may sometimes be similar, they may also differ significantly.

Is the Variation score limited to just the available action indicators?

While the action indicators are strong reflections of the overall subdimensions, they may not be fully exhaustive for every classroom. If a teacher performs an action that aligns well with the broader subdimension description but is not represented among the list of action indicators, the observer should clearly delineate in the Observation Notes (the second page of that subdimension's Scoring Sheets) how that teacher action 1) differs from the other action indicators, and 2) works toward fulfilling the subdimension description. This specific teacher action can then be considered a "new" action indicator for this specific observation, and its presence can then be integrated into an observer's considerations of how to score that subdimension's Variation.

How do I decide between two numbers within the same score category (i.e., how do I decide between a 3 or a 4?)?

Each scoring category (i.e., Early, Developing, and Advanced) has two affiliated numerical scores (1 or 2 for Early, 3 or 4 for Developing, 5 or 6 for Advanced). After deciding which category is most appropriate for an observed classroom, the observer will decide which of the two scores fits that classroom best using Table 03 below.

Table 03: Breakdown of Variation Score Categories

\begin{tabular}{|c|c|c|c|c|c|}
\hline \multicolumn{2}{|c|}{ Early } & \multicolumn{2}{|c|}{ Developing } & \multicolumn{2}{|c|}{ Advanced } \\
\hline 1 & 2 & 3 & 4 & 5 & 6 \\
\hline $\begin{array}{l}\text { None of the } \\
\text { subdimension's } \\
\text { action indicators } \\
\text { materialized } \\
\text { during the } \\
\text { observation. }\end{array}$ & $\begin{array}{l}\text { Only one of the } \\
\text { subdimension's } \\
\text { action indicators } \\
\text { materialized } \\
\text { during the } \\
\text { observation } \\
\text { (regardless of } \\
\text { frequency). }\end{array}$ & $\begin{array}{l}\text { Some (two or } \\
\text { more) of the } \\
\text { subdimension's } \\
\text { action indicators } \\
\text { materialized during } \\
\text { the observation, } \\
\text { one of which may } \\
\text { have appeared } \\
\text { multiple times. }\end{array}$ & $\begin{array}{l}\text { Some (two or more) } \\
\text { of the } \\
\text { subdimension's } \\
\text { action indicators } \\
\text { materialized during } \\
\text { the observation; } \\
\text { some action } \\
\text { indicators (but not } \\
\text { the majority) } \\
\text { appeared multiple } \\
\text { times. }\end{array}$ & $\begin{array}{l}\text { Most (i.e., the } \\
\text { majority) of the } \\
\text { subdimension's } \\
\text { action indicators } \\
\text { materialized during } \\
\text { the observation; } \\
\text { many of these } \\
\text { appeared multiple } \\
\text { times. }\end{array}$ & $\begin{array}{l}\text { Most (i.e., the } \\
\text { majority) of the } \\
\text { subdimension's } \\
\text { action indicators } \\
\text { materialized during } \\
\text { the observation; all } \\
\text { of these appeared } \\
\text { multiple times. }\end{array}$ \\
\hline
\end{tabular}

What do I do if I see no evidence for a particular subdimension in a classroom?

It is possible that none of a subdimension's action indicators materialize during the observation. In these circumstances, that subdimension should receive a Variation score of 1.

\section{Threats to Belonging:}

So far, this introduction has largely focused on teachers' actions - or the lack thereof - that facilitate students' sense of belonging inside mathematics classrooms. However, we recognize that there might be circumstances where teachers engage in actions that explicitly undermine or pose a threat to students' belonging. To allow observers to note and capture these instances in their scoring, we have created one final subdimension: Threats to Belonging.

This subdimension is structured similarly to the preceding seven subdimensions in that it contains Subdimension Details and Scoring Details, and provides space for Observation Notes. These three components of the subdimension provide information defining threats to belonging, instructions on how to score any threats to belonging that occur, and space to take notes on what is observed in the classroom related to this subdimension. However, this subdimension does NOT contain an Action Indicator Table. This table was excluded to prevent observers from spending too much time and attention attempting 
to locate threats to belonging. Instead, observers should focus more on the strengths that a teacher exhibits in the classroom as opposed to a teacher's deficits.

If and when a teacher engages in an action during the observation that directly undermines or poses a threat to students' belonging, observers should make note of these instances in this subdimension's Observation Notes. These notes should be detailed enough to allow the observer to sufficiently explain the instance without consuming too much time or attention.

It is important to note that there is a distinction between 1) the absence of belonging-related behaviors or missed opportunities to promote belonging, and 2) teacher behaviors that directly or explicitly undermine or pose a threat to students' belonging. The absence of belonging-related teacher actions or missed opportunities for them are already a consideration within the Quality and Variation scores of the other seven subdimensions, and they should NOT be considered again here. Instead, this subdimension captures teacher actions that moderately or overtly counter any of the seven subdimensions of a belonging-centered classroom. In other words, Threats to Belonging captures teacher actions that reject, dismiss, express insensitivity towards, disparage, stigmatize, or aggressively confront students or their physical, emotional, psychological, or academic needs.

Once the observation is completed and the other seven subdimensions have been scored, observers may score Threats to Belonging. Please note that the scoring for this subdimension is different from the other seven subdimensions. Instead of Quality and Variation, observers will be scoring Threats to Interpersonal Belonging and Threats to Instructional Belonging. Each of these two categories includes several action indicators, each of which reflects a threat to one of the other seven subdimensions of BCI (e.g., Threats to Safety to Be Wrong, Threats to Communal Orientation). Figure 06 below shows the Subdimension Details of Threats to Belonging, which categorizes and defines each of these action indicators.

\section{Figure 06: Scoring Details for Threats to Belonging}

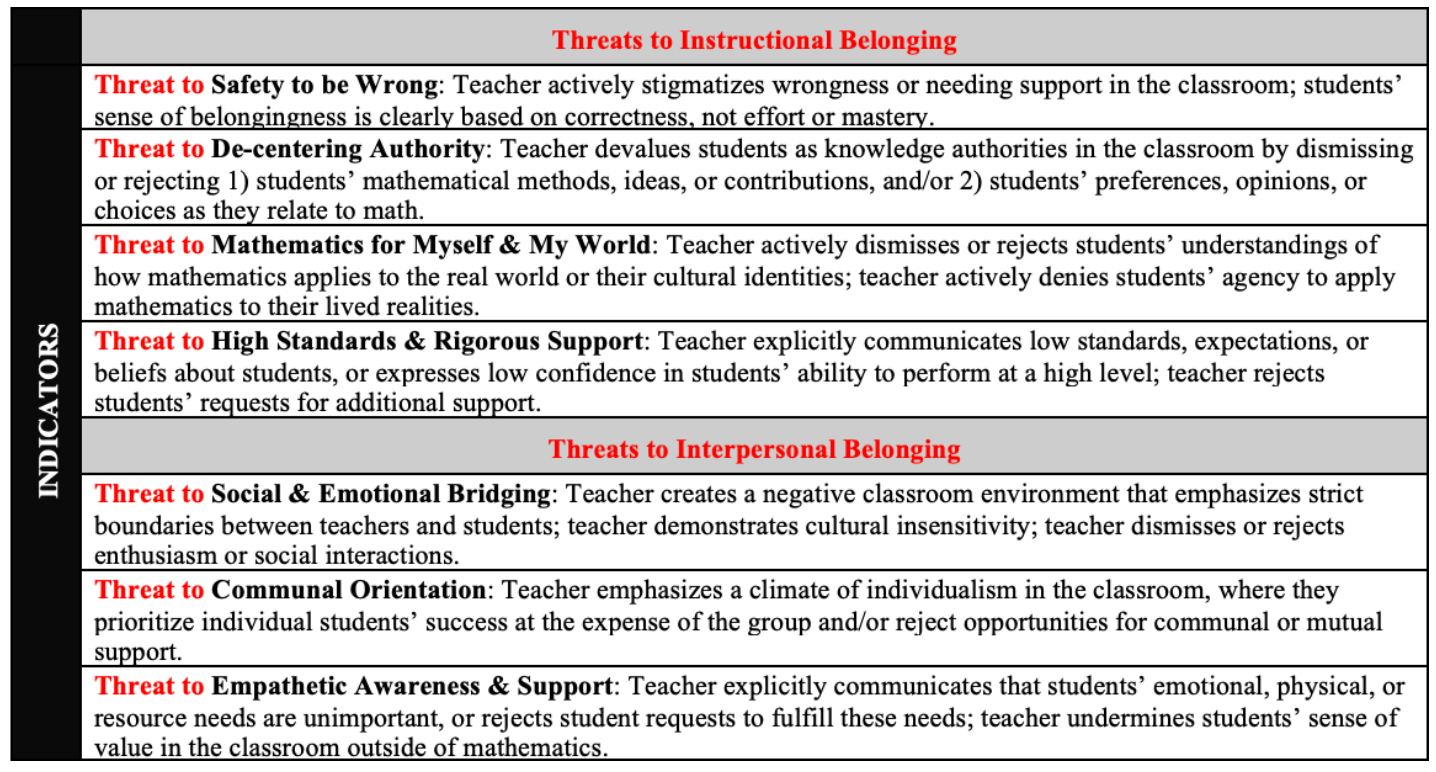

When scoring, each documented instance of a threat to belonging will be labeled according to the appropriate category (i.e., Threats to Instructional Belonging, Threats to Interpersonal Belonging). For example, if a Threat to Communal Orientation occurs, it will be categorized as a Threat to Interpersonal Belonging. Threats will then be classified as either moderate or overt (see Threats to Belonging section for details). Each threat will then be entered as a tally mark within the subdimension's scoring table according to its category and classification (see Figure 07 below). If no threats occur, one tally mark will be entered in the absent box. 
Figure 07: Scoring Table for Threats to Belonging

\begin{tabular}{|l|l|l|l|}
\hline \multicolumn{1}{|c|}{ Threats to Belonging } & Absent (0) & $\begin{array}{c}\text { Moderate Instances } \\
(-1)\end{array}$ & $\begin{array}{c}\text { Overt Instances } \\
(-2)\end{array}$ \\
\hline $\begin{array}{l}\text { Threats to Instructional Belonging: } \\
-\quad \text { Threat to Safety to be Wrong } \\
-\quad \text { Threat to De-centering Authority }\end{array}$ & & & \\
$-\quad \begin{array}{l}\text { Threat to Mathematics for Myself \& My World } \\
-\quad \text { Threat to High Standards \& Rigorous Support }\end{array}$ & & & \\
\hline $\begin{array}{l}\text { Threats to Interpersonal Belonging: } \\
-\quad \text { Threat to Social \& Emotional Bridging } \\
-\quad \text { Threat to Communal Orientation } \\
-\quad \text { Threat to Empathetic Awareness \& Support }\end{array}$ & & & \\
\hline
\end{tabular}

Each Threats to Belonging category will then receive its own score based on where the majority of tally marks fell. For example, if there were three tally marks under Moderate Instances of Threats to Interpersonal Belonging but only one tally mark under Overt Instances, then Threats to Interpersonal Belonging would receive a score of - 1 . Conversely, if there were three tally marks under Overt Instances of Threats to Interpersonal Belonging but only one tally mark under Moderate Instances, then Threats to Interpersonal Belonging would receive a score of -2. Finally, if the only tally mark present for Threats to Interpersonal Belonging fell under the Absent classification, then Threats to Interpersonal Belonging would receive a score of 0 . 


\section{Quick Start Guide to Observational Scoring}

Observing and scoring a classroom performance can be challenging, especially when doing so in real time. Before observing any classroom with this protocol, you should spend at least thirty minutes reading these Quick Start Guide pages and studying the subdimension materials below. This study period is very important, because it is unlikely that you will be able to consult these materials closely once you begin observing. It is also important to understand that this observational scoring proceeds in two consecutive phases:

The Observation phase is when you are observing what the teacher is doing in the classroom.

The Scoring phase occurs later, when you are applying a score to evaluate what a teacher did in the classroom.

If you are not experienced with observational scoring or using this protocol, it is important to keep these two phases separate: observe first and score second. Trying to observe and score at the same time will likely result in errors, such as missing important events in the classroom or misusing the scoring system. Once you have become more experienced and comfortable with the protocol, some coders may naturally begin to observe and score at the same time. However, for simplicity and accuracy, we encourage most coders to observe first then score second.

\section{What should I be paying attention to while I am OBSERVING?}

While observing a classroom, there are two main things you should be paying to: 1) teacher actions (what the teacher is doing in the classroom), and 2) the overall description of the subdimension (how the teacher's actions fit the categories at the top of the scoring sheet for each subdimension). The goal is to evaluate the alignment between the teacher's actions and the subdimension descriptions. While observing, take short notes on teacher actions that stand out to you and seem belonging-relevant. At the same time, examine the subdimension descriptions periodically. If possible, avoid scoring while you are observing. Your attention should be focused on what the teacher is doing across the multiple subdimension descriptions.

\section{What should I be paying attention to while I am SCORING?}

After you finish the observation, you should begin paying closer attention to the action indicators (listed underneath each subdimension description). These action indicators will help you evaluate the alignment between the teacher's actions and the overall description of each subdimension. Teachers who showed high-quality behaviors similar to what the action indicators describe are more likely to receive higher subdimension scores. Teachers who did not show behaviors consistent with what the actions indicators describe or who did so in superficial ways are more likely to receive lower subdimension scores. You should use your observation notes to determine how the teacher's actions relate to the action indicators. Based on those notes and your personal evaluation, you will provide a Quality score and Variation score for each subdimension (see more details on Quality and Variation below).

Note 1. As you become more familiar with the action indicators, you may begin to notice them immediately during the observation phase. If that happens naturally, feel free to take notes on those action indicators during the observation phase. However, be careful not to let a narrow focus on the action indicators distract you from what the teacher is doing and the subdimension descriptions during observation.

Note 2. The action indicators are strong reflections of the overall subdimension, but they may not be fully exhaustive for every classroom. In some circumstances, a teacher may have done something that reflects the overall subdimension very well, but is not represented among the action indicators. In those infrequent circumstances, the observer should note the teacher's behavior in the notes section with clear detail explaining the nature of the unique action. After doing so, the observer may use that teacher behavior as an action indicator in their scoring.

\section{Are all Action Indicators Weighted or Interpreted Equally?}

This question is complex, but the simple answer is NO. Classrooms can differ in many ways; therefore, expect some action indicators will occur more often than others. Individual teachers' performance of action indicators may also differ in meaningfulness or quality (i.e., teachers may be "early" on some actions but may be "advanced" on others). Therefore, we encourage observers to consider each action indicator within its unique context, and to consider how the teacher's enactment of that action indicator but a subdimension's Quality or Variation for that specific classroom. The distinctions between scoring Quality and Variation are outlined below. 


\section{How do I score Quality?}

Quality refers to the degree to which a teacher's actions fit the subdimension description. An important consideration for Quality is the action indicators for each subdimension, because their presence and strength will help an observer evaluate to what degree the description has been fulfilled. To assist with this, we have provided "Scoring Support" pages for each subdimension that include tables with concrete- but not exhaustive - examples of action indicators from real classrooms. The examples in these tables have been given Early, Developing, and Advanced labels, not unlike the Quality scores on the scoring sheets. However, remember that you are scoring the overall subdimension, not the action indicators. While it might be tempting to use the example tables to score the action indicators, these tables are intended as guides to show how action indicators might be enacted differently across classrooms. It is helpful to take a broad view of Quality while scoring, focusing less on individual action indicators and more on how teachers' actions meaningfully align with the action indicators and cumulatively build the subdimension description.

It is also important to remember that Quality refers to consistency of Quality. In general, teachers whose actions consistently align with the indicators in "rich," "significant," or "meaningful" ways are more likely to receive an Advanced Quality score for that subdimension. Teachers whose actions consistently align with the indicators in "superficial," "partial," or "inauthentic" ways are more likely to receive an Early Quality score for the subdimension. Teachers whose actions are inconsistent (i.e., sometimes "superficial" and sometimes "meaningful") or consistently fall somewhere between "superficial" and "meaningful" (i.e., of moderate strength) are more likely to receive a Developing Quality score for the subdimension. However, consistently does not mean exclusively; even teachers that receive an Advanced score are likely to perform superficial actions occasionally.

\section{How do I score Variation?}

Variation refers to the number of distinct action indicators that a teacher performs throughout the observation and their frequency. Variation describes when teachers perform a range of different actions, regardless of the quality of each action. Variation may be especially important in diverse classrooms where a range of different actions may be necessary to meet the unique needs of different students within the class.

Scoring Variation requires observers to identify how many different action indicators were present during the observation and how often they were enacted, regardless of the quality or substance of those indicators. The broader the range and the higher the frequency of action indicators present, the higher the Variation score. Please note that a teacher does not have to perform all of the action indicators to receive an Advanced Variation score. It is unlikely that a teacher will perform all of the action indicators within a particular subdimension during a single observational period.

\section{What if I see teacher actions that work against belonging in the classroom?}

Teacher actions that directly or explicitly pose a threat to students' belonging are catalogued as Threats to Belonging. This final subdimension extends beyond the absence of belonging-related behaviors or missed opportunities to promote belonging (which are already a consideration within the Quality and Variation scores of the seven subdimensions), and instead reflects teacher actions that moderately or overtly counter the seven subdimensions of a belonging-centered classroom.

Observers should note any explicit threats to belonging that materialize during the observation. Once the observation is completed, observers will categorize each instance as a Threat to Interpersonal Belonging or a Threat to Instructional Belonging, and then classify each instance as Moderate (i.e., teacher rejects, dismisses, or expresses insensitivity towards students or their needs) or as Overt (i.e., teacher disparages, stigmatizes, or aggressively confronts students or their needs). Based on the category and classification, each instance will then be entered as a tally mark within the subdimension's scoring table. If no threats to belonging materialize, one tally mark will be entered in the Absent box.

If the majority of tally marks for a category (i.e., Threats to Interpersonal Belonging or Threats to Instructional Belonging) were classified as Overt, that category will receive a score of -2. If the majority of tally marks for a category were classified as Moderate, that category will receive a score of -1 . If no threats to belonging materialized for a category (i.e., the tally mark is in the Absent box), that category will receive a score of 0 . 


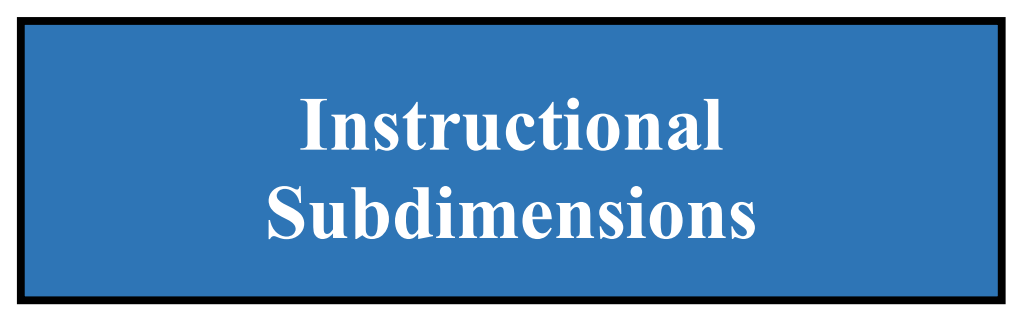




\section{SAFETY TO BE WRONG}

Teacher creates a space where students do not feel stigmatized for wrongness or needing support. Teacher disarms and normalizes wrongness. Students have the opportunity to break the pattern of judging their mathematical belongingness simply based on correctness. Mastery and effort are celebrated over performance and talent.

Normalizing Difficulties: Teacher shows that wrongness is a normal and expected part of the learning process.

Creating Space for Questions: Teacher seeks out questions from students, creating an environment that is a safe place for uncertainty and learning.

Task-focused Encouragement: Teacher attempts to disarm self-doubt and encourages students to persist through difficult mathematical tasks.

Celebrating Mastery-focused Behavior: Teacher celebrates students' effort or strategies as examples of how to overcome difficulties on the road to mastery.

Modeling Mistakes for Learning: Teacher models their own or students' mistakes as potential teaching moments for students. Teacher acknowledges mistakes/errors and uses them to create opportunities for learning.

\section{Scoring Details:}

Quality: The extent to which the observable action indicators exemplify the Safety to Be Wrong description (listed above). Low-quality examples may reflect the subdimension description but only do so partially, superficially, or inauthentically. Highquality examples reflect the subdimension description in significant, rich, and meaningful ways, or in some instances may exceed the subdimension description.

Variation: Indicates the range/quantity of different action indicators that were present throughout the observation cycle. The more indicators observed, the higher the observation score. Note: It is unlikely a teacher will be able cover the full breadth of action indicators in a single class meeting.

Scoring: Quality and Variation should be evaluated and scored independently. Select the level of development that best represents the teacher's actions throughout the classroom observation. Within the selected level of development, decide whether the teacher's actions fall on the lower or higher end and select the relevant number.

\begin{tabular}{|c|c|c|c|}
\hline & $\begin{array}{l}\text { Early } \\
(1,2)\end{array}$ & $\begin{array}{l}\text { Developing } \\
\quad(3,4)\end{array}$ & $\begin{array}{l}\text { Advanced } \\
\quad(5,6)\end{array}$ \\
\hline Quality & $\begin{array}{c}\text { The quality of teacher actions } \\
\text { consistently reflects Safety to Be } \\
\text { Wrong in a shallow, partial, } \\
\text { light, or inauthentic way }\end{array}$ & $\begin{array}{l}\text { The quality of teacher actions is } \\
\text { mixed/inconsistent or reflects } \\
\text { Safety to Be Wrong in a way that } \\
\text { falls between superficial and } \\
\text { meaningful }\end{array}$ & $\begin{array}{c}\text { The quality of teacher actions } \\
\text { consistently reflects Safety to Be } \\
\text { Wrong in a rich, significant, or } \\
\text { enduring way }\end{array}$ \\
\hline Score: & & & \\
\hline Variation & $\begin{array}{l}\text { Teacher engages none or one of } \\
\text { the action indicators }\end{array}$ & $\begin{array}{l}\text { Teacher engages two or more of } \\
\text { the action indicators }\end{array}$ & $\begin{array}{l}\text { Teacher engages most of the } \\
\text { action indicators multiple times }\end{array}$ \\
\hline Score: & & & \\
\hline
\end{tabular}




\begin{tabular}{|l|l|l|}
\hline Date: & Observer: & Classroom: \\
\hline Teacher: & Grade: & Topic: \\
\hline \multicolumn{1}{|c|}{ Observation Notes: }
\end{tabular}




\section{Action Indicator Table for Safety to Be Wrong}

To support your scoring, here we provide some specific examples from real classrooms for each action indicator and its varying levels of quality.

However, we strongly advise using these examples only as guideposts. The action indicators can materialize in a wide variety of ways, and the

examples provided here are not fully comprehensive or exhaustive.

\begin{tabular}{|c|c|c|c|c|}
\hline \multicolumn{5}{|c|}{ Levels of Quality for Safety to be Wrong Action Indicators } \\
\hline Action Indicators & & Early & Developing & Advanced \\
\hline & 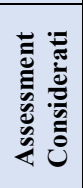 & $\begin{array}{l}\text { Teacher engages in one or } \\
\text { more behaviors in a brief } \\
\text { and/or superficial way. }\end{array}$ & $\begin{array}{l}\text { Teacher 1) engages in one behavior in a } \\
\text { meaningful way and the others not at all } \\
\text { or superficially, or 2) engages in two or } \\
\text { more behaviors in a way that falls } \\
\text { between superficial and meaningful. }\end{array}$ & $\begin{array}{l}\text { Teacher engages in two or more behaviors in an extended } \\
\text { and/or meaningful way. }\end{array}$ \\
\hline $\begin{array}{ll}\text { Normalizing Difficulties: } \\
- & \text { Shows errors are normal. } \\
- & \text { Attempts to remove negative } \\
\text { emotions associated w/ errors. } \\
\text { - } \\
\quad \text { Shows wrongness is part of } \\
\text { learning. }\end{array}$ & \multirow{5}{*}{ 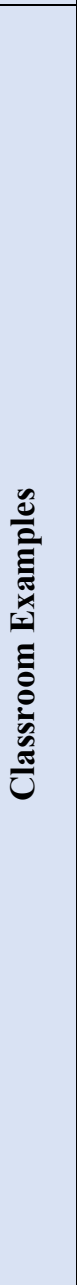 } & $\begin{array}{l}\text { When a student provides an } \\
\text { incorrect answer, the teacher } \\
\text { responds, "That's okay!" } \\
\text { before moving on to another } \\
\text { student. }\end{array}$ & $\begin{array}{l}\text { When a student provides an incorrect } \\
\text { answer, the teacher responds, "That's } \\
\text { fine! We all make mistakes sometimes. } \\
\text { Now, let's fix it!" }\end{array}$ & $\begin{array}{l}\text { When having students correct homework, the teacher says, } \\
\text { "If you made a mistake, don't stress it. It's the corrections } \\
\text { that are important. Corrections help you identify what you } \\
\text { did wrong and how you can do better next time." }\end{array}$ \\
\hline $\begin{array}{ll}\text { Creating Space for Questions: } \\
-\quad \text { Invites student questions. } \\
-\quad \text { Creates space to respond to } \\
\text { student questions. } \\
\text { Creates safe environment for } \\
\text { questioning. }\end{array}$ & & $\begin{array}{l}\text { At the end of the lesson, the } \\
\text { teacher pauses briefly to ask, } \\
\text { "Any questions?" then } \\
\text { launches into an explanation } \\
\text { of the homework. }\end{array}$ & $\begin{array}{l}\text { During the lesson, a student raises their } \\
\text { hand. The teacher stops the instruction, } \\
\text { encourages the student to ask the } \\
\text { question, and then spends several minutes } \\
\text { clarifying the question for them. }\end{array}$ & $\begin{array}{l}\text { After the first part of the lesson, the teacher says, "I know } \\
\text { that might have been a little confusing, so I want anyone } \\
\text { with a question to ask it," which normalizes inquiry for } \\
\text { students. After spending extended time responding to a } \\
\text { student's question, the teacher thanks them for helping to } \\
\text { clarify that issue for the entire class. }\end{array}$ \\
\hline $\begin{array}{l}\text { Task-focused Encouragement: } \\
-\quad \text { Disarms self-doubt. } \\
\text { Encourages students to } \\
\text { persist. } \\
\text { Explains the importance of } \\
\text { persisting. }\end{array}$ & & $\begin{array}{l}\text { A student calls out to the } \\
\text { teacher, "This is hard!" The } \\
\text { teacher responds, "Keep } \\
\text { going! You got this!" }\end{array}$ & $\begin{array}{l}\text { A student is clearly struggling in their } \\
\text { independent work. The teacher } \\
\text { approaches the student and says, "If } \\
\text { you're having trouble with this one, } \\
\text { move on to the next one. But think! } \\
\text { Because you really can do this!" }\end{array}$ & $\begin{array}{l}\text { A student expresses that they're struggling with } \\
\text { reciprocals. The teacher replies, "We haven't talked about } \\
\text { reciprocals this week, so you may want to look at your } \\
\text { notes to see how that ties into the inverse operation. If } \\
\text { you're not sure, it's always important to try, otherwise } \\
\text { you'll never learn!" }\end{array}$ \\
\hline $\begin{array}{l}\text { Celebrating Mastery- } \\
\text { focused Behavior: } \\
\text { - } \quad \text { Acknowledges student } \\
\text { process and persistence. } \\
\text { - } \quad \text { Celebrates effort or strategy } \\
\text { above answers. } \\
\text { - } \quad \text { Uses students' processes or } \\
\text { persistence as model for class. }\end{array}$ & & $\begin{array}{l}\text { A student answers a question } \\
\text { incorrectly, and the teacher } \\
\text { responds, "Good effort," } \\
\text { before moving onto another } \\
\text { student. }\end{array}$ & $\begin{array}{l}\text { A student makes a mistake when showing } \\
\text { their work, but notices and fixes it. The } \\
\text { teacher responds, "Very good! I like that } \\
\text { you fixed that so quickly! Thank you!" }\end{array}$ & $\begin{array}{l}\text { A student finishes showing her work at the board, but the } \\
\text { answer is incorrect. The teacher responds, "Let me tell you } \\
\text { what I like that she did. I like how she went through the } \\
\text { process step by step. I like that she would strike out when } \\
\text { she's finished using that number. She put a line through it. } \\
\text { But there's something she forgot to do. She's carrying } \\
\text { numbers and leaving them out there. Anybody see that?" }\end{array}$ \\
\hline $\begin{array}{l}\text { Modeling Mistakes for Learning: } \\
-\quad \text { Acknowledges own } \\
\text { limitations or mistakes. } \\
\text { - Uses mistakes (students' or } \\
\text { own) as teaching moments. } \\
\text { Incorporates students into the } \\
\text { process of fixing mistakes. }\end{array}$ & & $\begin{array}{l}\text { A teacher makes a mistake } \\
\text { while walking students } \\
\text { through a problem. The } \\
\text { teacher says, "Oops, I made } \\
\text { a mistake here." He then } \\
\text { fixes it quickly and moves } \\
\text { forward with the problem. }\end{array}$ & $\begin{array}{l}\text { A student makes a mistake while solving } \\
\text { a problem at the board. Another student } \\
\text { points out that a mistake was made, to } \\
\text { which the teacher responds, "Okay, } \\
\text { what's wrong?" The student identifies the } \\
\text { mistake, and the teacher says, "Good!" }\end{array}$ & $\begin{array}{l}\text { A teacher and student finish working through a problem at } \\
\text { the board. Another student at his desk points out that the } \\
\text { answer is incorrect. The teacher asks, "What did we do } \\
\text { wrong?" and encourages the student at his desk to walk the } \\
\text { student at the board through rectifying the problem. When } \\
\text { finished, the teacher says, "I love when you help each } \\
\text { other understand! Beautiful!" }\end{array}$ \\
\hline
\end{tabular}




\section{Equity Statement for Safety to Be Wrong:}

In traditional mathematics classrooms, teachers often foreground correctness and prioritize the final product over mathematical process. This can alienate students, who might feel that their belongingness in math is based on their ability to produce the "right" answer or might feel threatened by the stigma associated with being "wrong." The Safety to Be Wrong subdimension attempts to address these concerns. For example, when teachers "Normalize Difficulties," they destigmatize "wrongness" by communicating to students that "wrongness" is expected and can contribute to the learning process. Similarly, when teachers "Celebrate Mastery-focused Behavior," they recognize and affirm student process as opposed to student product, which demonstrates that students' mathematical thinking is valued more than their correctness (Patrick et al., 2011). When teachers engage in behaviors that communicate to students that it is "safe to be wrong," they can remove the threat and stigma that students often encounter in mathematics. This can promote belongingness by reducing alienation and by inviting more equitable participation from students in the math classroom (Gutiérrez, 2012; Kozlowski \& Si, 2019). 


\section{References}

Adair, J. K., \& Pastori, G. (2011). Developing qualitative coding frameworks for educational research: Immigration, education, and the Children Crossing Borders project. International Journal of Research and Method in Education, 34(1), 31-47. https://doi.org/10.1080/1743727X.2011.552310

Adelabu, D. H. (2007). Time perspective and school membership as correlates to academic achievement among African American adolescents. Adolescence, 42(167), 525-538.

Allen, K., Kern, M. L., Vella-Brodrick, D., Hattie, J., \& Waters, L. (2018). What schools need to know about fostering school belonging: A meta-analysis. Educational Psychology Review, 30(1), 1-34. https://doi.org/10.1007/s10648016-9389-8

Anderman, L. H. (2003). Academic and social perceptions as predictors of change in middle school students' sense of school belonging. The Journal of Experimental Education, 72(1), 5-22.

https://doi.org/10.1080/00220970309600877

Anderson-Butcher, D., Amorose, A., Iachini, A., \& Ball, A. (2012). The development of the Perceived School Experiences Scale. Research on Social Work Practice, 22(2), 186-194. https://doi.org/10.1177/1049731511419866

Bartell, T. G. (2013). Learning to teach mathematics for social justice: Negotiating social justice and mathematical goals. Journal for Research in Mathematics Education, 44(1), 129-163. https://doi.org/10.5951/jresematheduc.44.1.0129

Bartolomé, L. I. (1994). Beyond the methods fetish: Toward a humanizing pedagogy. Harvard Educational Review, 64(2), 173-194. https://doi.org/10.17763/haer.64.2.58q5m5744t325730

Battey, D. (2013). Access to mathematics: “A possessive investment in Whiteness.” Curriculum Inquiry, 43(3), 332-359. https://doi.org/10.1111/curi.12015

Baumeister, R. F., \& Leary, M. R. (1995). The need to belong: Desire for interpersonal attachments as a fundamental human motivation. Psychological Bulletin, 117(3), 497-529. https://doi.org/10.1037/0033-2909.117.3.497

Beechum, N. W. (2020). Expanding visions of success in mathematics for marginalized students: Building more equitable and inclusive mathematics environments. Student Experience Research Network. http://studentexperiencenetwork.org/wp-content/uploads/2020/06/Williams-Beechum-Expanding-Visions.pdf

Blau, J. R. (2003). Race in the schools: Perpetuating White dominance? Lynne Rienner Publishers.

Booker, K. C. (2004). Exploring school belonging and academic achievement in African American adolescents. Curriculum and Teaching Dialogue, 6(2), 131-143.

Booker, K. C. (2007). Likeness, comfort, and tolerance: Examining African American adolescents' sense of school belonging. The Urban Review, 39(3), 301-317. https://doi.org/10.1007/s11256-007-0053-y

Boston, C., \& Warren, S. R. (2017). The effects of belonging and racial identity on urban African American high school students' achievement. Journal of Urban Learning, Teaching, and Research, 13, 26-33.

Bottiani, J. H., Bradshaw, C. P., \& Mendelson, T. (2017). A multilevel examination of racial disparities in high school discipline: Black and White adolescents' perceived equity, school belonging, and adjustment problems. Journal of Educational Psychology, 109(4), 532-545. https://doi.org/10.1037/edu0000155

Boykin, A. W., \& Bailey, C. T. (2000). The role of cultural factors in school relevant cognitive functioning: Synthesis of findings on cultural contexts, cultural orientations, and individual differences (Report No. 42). Center for Research on the Education of Students Placed at Risk (CRESPAR). https://files.eric.ed.gov/fulltext/ED441880.pdf

Boykin, A. W., \& Ellison, C. M. (1995). The multiple ecologies of Black youth socialization: An Afrographic analysis. In R. L. Taylor (Ed.), African American youth: Their social and economic status in the United States (pp. 93-128). Praeger.

Boykin, A. W., Jagers, R. J., Ellison, C. M., \& Albury, A. (1997). Communalism: Conceptualization and measurement of an Afrocultural social orientation. Journal of Black Studies, 27(3), 409-418. https://doi.org/10.1177/002193479702700308

Brown, K. D., \& Brown, A. L. (2010). Silenced memories: An examination of the sociocultural knowledge on race and racial violence in official school curriculum. Equity \& Excellence in Education, 43(2), 139-154. https://doi.org/10.1080/10665681003719590

Bullock, E. C. (2017). Only STEM can save us? Examining race, place, and STEM education as property. Educational Studies, 53(6), 628-641. https://doi.org/10.1080/00131946.2017.1369082 
Cook, J. E., Purdie-Vaughns, V., Garcia, J., \& Cohen, G. L. (2012). Chronic threat and contingent belonging: Protective benefits of values affirmation on identity development. Journal of Personality and Social Psychology, 102(3), 479-496. https://doi.org/10.1037/a0026312

Coolican, H. (2018). Research methods and statistics in psychology (7th ed.). Routledge.

Darling-Hammond, L. (2013). Inequality and school resources: What it will take to close the opportunity gap. In P. L. Carter \& K. G. Welner (Eds.), Closing the opportunity gap: What America must do to give every child an even chance (pp. 77-97). Oxford University Press.

Deci, E. L., Vallerand, R. J., Pelletier, L. G., \& Ryan, R. M. (1991). Motivation and education: The self-determination perspective. Educational Psychologist, 26(3-4), 325-346. https://doi.org/10.1080/00461520.1991.9653137

Delgado, M. Y., Ettekal, A. V., Simpkins, S. D., \& Schaefer, D. R. (2016). How do my friends matter? Examining Latino adolescents' friendships, school belonging, and academic achievement. Journal of Youth and Adolescence, 45(6), 1110-1125. https://doi.org/10.1007/s10964-015-0341-X

Diekman, A. B., Steinberg, M., Brown, E. R., Belanger, A. L., \& Clark, E. K. (2017). A goal congruity model of role entry, engagement, and exit: Understanding communal goal processes in STEM gender gaps. Personality and Social Psychology Review, 21(2), 142-175. https://doi.org/10.1177/1088868316642141

Diversity in Mathematics Education Center for Learning and Teaching. (2007). Culture, race, power, and mathematics education. In F. K. Lester (Ed.), Second handbook of research on mathematics teaching and learning (pp. 405-433). Information Age Publishing.

Dotterer, A. M., \& Lowe, K. (2011). Classroom context, school engagement, and academic achievement in early adolescence. Journal of Youth and Adolescence, 40(12), 1649-1660. https://doi.org/10.1007/s10964-011-9647-5

Dotterer, A. M., McHale, S. M., \& Crouter, A. C. (2009). Sociocultural factors and school engagement among African American youth: The roles of racial discrimination, racial socialization, and ethnic identity. Applied Developmental Science, 13(2), 61-73. https://doi.org/10.1080/10888690902801442

Dweck, C. S. (1986). Motivational processes affecting learning. American Psychologist, 41(10), 10401048. https://doi.org/10.1037/0003-066X.41.10.1040

Eccles, J. S., \& Midgley, C. (1989). Stage/environment fit: Developmentally appropriate classrooms for early adolescents. In R. Ames \& C. Ames (Eds.), Research on motivation in education (Vol. 3, pp. 139-181). Academic Press.

Eccles, J. S., Midgley, C., Wigfield, A., \& Buchanan, C. (1993). Development during adolescence: The impact of stageenvironment fit on young adolescents' experiences in schools and in families. American Psychologist, 48(2), 90 101. https://doi.org/10.1037/0003-066X.48.2.90

Eccles, J. S., \& Roeser, R. W. (2011). School and community influences on human development. In M. H. Bornstein \& M. E. Lamb (Eds.), Developmental science: An advanced textbook (pp. 571-643). Psychology Press.

Ellerbrock, C., Kiefer, S. M., \& Alley, K. M. (2014). School-based interpersonal relationships: Setting the foundation for young adolescents' belonging in middle school. Middle Grades Research Journal, 9(2), 1-17.

Emdin, C. (2010). Affiliation and alienation: Hip-hop, rap, and urban science education. Journal of Curriculum Studies, 42(1), 1-25. https://doi.org/10.1080/00220270903161118

Emdin, C. (2017). For White folks who teach in the hood... and the rest of y'all too: Reality pedagogy and urban education. Beacon Press.

Erickson, F. (2006). Definition and analysis of data from videotape: Some research procedures and their rationales. In J. L. Green, G. Camilli, P. B. Elmore, A. Skukauskaité, \& E. Grace (Eds.), Handbook of complementary methods in education research (pp. 177-191). Lawrence Erlbaum Associates.

Faircloth, B. S., \& Hamm, J. V. (2005). Sense of belonging among high school students representing four ethnic groups. Journal of Youth and Adolescence, 34(4), 293-309. https://doi.org/10.1007/s10964-005-5752-7

Ferguson, R. (2012). Can student surveys measure teaching quality? Phi Delta Kappan, 94(3), 24-28. https://doi.org/10.2307/41763671

Ferguson, R. F., Ludwig, J., \& Rich, W. (2001). A diagnostic analysis of Black-White GPA disparities in Shaker Heights, Ohio. Brookings Papers on Education Policy, 2001, 347-414. https://doi.org/10.1353/pep.2001.0003

Ford, A. C., \& Sassi, K. (2014). Authority in cross-racial teaching and learning: (Re)considering the transferability of warm demander approaches. Urban Education, 49(1), 39-74. https://doi.org/10.1177/0042085912464790

Frankenberg, E. (2013). The role of residential segregation in contemporary school segregation. Education and Urban Society, 45(5), 548-570. https://doi.org/10.1177/0013124513486288 
Freeman, T. M., Anderman, L. H., \& Jensen, J. M. (2007). Sense of belonging in college freshmen at the classroom and campus levels. The Journal of Experimental Education, 75(3), 203-220. https://doi.org/10.3200/JEXE.75.3.203220

Furrer, C., \& Skinner, E. (2003). Sense of relatedness as a factor in children's academic engagement and performance. Journal of Educational Psychology, 95(1), 148-162. https://doi.org/10.1037/0022-0663.95.1.148

Garcia-Reid, P. (2007). Examining social capital as a mechanism for improving school engagement among low income Hispanic girls. Youth \& Society, 39(2), 164-181. https://doi.org/10.1177/0044118X07303263

Gay, G. (2010). Culturally responsive teaching: Theory, research, and practice (2nd ed.). Teachers College Press.

Gillen-O'Neel, C., \& Fuligni, A. (2013). A longitudinal study of school belonging and academic motivation across high school. Child Development, 84(2), 678-692. https://doi.org/10.1111/j.1467-8624.2012.01862.x

Goodenow, C. (1993). Classroom belonging among early adolescent students: Relationships to motivation and achievement. The Journal of Early Adolescence, 13(1), 21-43. https://doi.org/10.1177/0272431693013001002

Goodenow, C., \& Grady, K. E. (1993). The relationship of school belonging and friends' values to academic motivation among urban adolescent students. The Journal of Experimental Education, 62(1), 60-71. https://doi.org/10.1080/00220973.1993.9943831

Gray, D. L., Hope, E. C., \& Matthews, J. S. (2018). Black and belonging at school: A case for interpersonal, instructional, and institutional opportunity structures. Educational Psychologist, 53(2), 97-113. https://doi.org/10.1080/00461520.2017.1421466

Green, M., Emery, A., Sanders, M., \& Anderman, L. H. (2016). Another path to belonging: A case study of middle school students' perspectives. The Educational and Developmental Psychologist, 33(1), 85-96. https://doi.org/10.1017/edp.2016.4

Gutiérrez, R. (2012). Context matters: How should we conceptualize equity in mathematics education? In B. HerbelEisenmann, J. Choppin, D. Wagner, \& D. Pimm (Eds.), Equity in discourse for mathematics education (pp. 1733). Springer.

Gutiérrez, R. (2018). When mathematics teacher educators come under attack. Mathematics Teacher Educator, 6(2), 6874. https://doi.org/10.5951/mathteaceduc.6.2.0068

Gutstein, E., Lipman, P., Hernandez, P., \& de los Reyes, R. (1997). Culturally relevant mathematics teaching in a Mexican American context. Journal for Research in Mathematics Education, 28(6), 709-737. https://doi.org/10.2307/749639

Hamm, J. V., \& Faircloth, B. S. (2005). Peer context of mathematics classroom belonging in early adolescence. The Journal of Early Adolescence, 25(3), 345-366. https://doi.org/10.1177/0272431605276932

Headland, T. N., Pike, K. L., \& Harris, M. (Eds.). (1990). Emics and etics: The insider/outsider debate. Sage Publications.

Henry, K. L., Swaim, R. C., \& Slater, M. D. (2005). Intraindividual variability of school bonding and adolescents' beliefs about the effect of substance use on future aspirations. Prevention Science, 6(2), 101-112. https://doi.org/10.1007/s11121-005-3409-0

Hidi, S. (2000). An interest researcher's perspective: The effects of extrinsic and intrinsic factors on motivation. In C. Sansone \& J. M. Harackiewicz (Eds.), Intrinsic and extrinsic motivation: The search for optimal motivation and performance (pp. 309-339). Academic Press. https://doi.org/10.1016/B978-012619070-0/50033-7

Hill, L. G., \& Werner, N. E. (2006). Affiliative motivation, school attachment, and aggression in school. Psychology in the Schools, 43(2), 231-246. https://doi.org/10.1002/pits.20140

Huang, C. Y., \& Stormshak, E. A. (2011). A longitudinal examination of early adolescence ethnic identity trajectories. Cultural Diversity and Ethnic Minority Psychology, 17(3), 261270. https://doi.org/10.1037/a0023882

Hughes, J. N. (2011). Longitudinal effects of teacher and student perceptions of teacher-student relationship qualities on academic adjustment. The Elementary School Journal, 112(1), 38-60. https://doi.org/10.1086/660686

Hughes, J. N., Im, M. H., \& Allee, P. J. (2015). Effect of school belonging trajectories in grades 6-8 on achievement: Gender and ethnic differences. Journal of School Psychology, 53(6), 493-507.

https://doi.org/10.1016/i.jsp.2015.08.001

Juvonen, J. (2006). Sense of belonging, social relationships, and school functioning. In P. A. Alexander \& P. H. Winne (Eds.), Handbook of educational psychology (2nd ed., pp. 655-674). Erlbaum. 
Kiefer, S. M., Alley, K. M., \& Ellerbrock, C. R. (2015). Teacher and peer support for young adolescents' motivation, engagement, and school belonging. RMLE Online, 38(8), 1-18. https://doi.org/10.1080/19404476.2015.11641184

King, J. E., \& Swartz, E. E. (2016). The Afrocentric praxis of teaching for freedom: Connecting culture to learning. Routledge.

Kirkland, D. E., \& Jackson, A. (2009). "We real cool": Toward a theory of Black masculine literacies. Reading Research Quarterly, 44(3), 278-297. https://doi.org/10.1598/RRQ.44.3.3

Klugman, J. (2013). The Advanced Placement arms race and the reproduction of educational inequality. Teachers College Record, 115(5).

Kogachi, K., \& Graham, S. (2020). Numerical minority status in middle school and racial/ethnic segregation in academic classes. Child Development, 91(6), 1-20. https://doi.org/10.1111/cdev.13408

Koo, T. K., \& Li, M. Y. (2016). A guideline of selecting and reporting intraclass correlation coefficients for reliability research. Journal of Chiropractic Medicine, 15(2), 155-163. https://doi.org/10.1016/j.jcm.2016.02.012

Kozlowski, J. S., \& Si, S. (2019). Mathematical creativity: A vehicle to foster equity. Thinking Skills and Creativity, 33, 1-8. https://doi.org/10.1016/j.tsc.2019.100579

Ladson-Billings, G. (1995). Toward a theory of culturally relevant pedagogy. American Educational Research Journal, 32(3), 465-491. https://doi.org/10.3102/00028312032003465

Libbey, H. P. (2004). Measuring student relationships to school: Attachment, bonding, connectedness, and engagement. The Journal of School Health, 74(7), 274-283. https://doi.org/10.1111/j.1746-1561.2004.tb08284.x

Linnakylä, P. \& Malin, A. (2008). Finnish students' school engagement profiles in the light of PISA 2003. Scandanavian Journal of Educational Research, 52(6), 583-602. https://doi.org/10.1080/00313830802497174

Malkus, N. (2016). AP at scale: Public school students in Advanced Placement, 1990-2013. American Enterprise Institute. https://www.aei.org/research-products/report/ap-at-scale-public-school-students-in-advanced-placement1990-2013/

Maloney, T., \& Matthews, J. S. (2020). Teacher care and students' sense of connectedness in the urban mathematics classroom. Journal for Research in Mathematics Education, 51(4), 399-432. https://doi.org/10.5951/jresematheduc-2020-0044

Martin, A. J., \& Dowson, M. (2009). Interpersonal relationships, motivation, engagement, and achievement: Yields for theory, current issues, and educational practice. Review of Educational Research, 79(1), 327-365. https://doi.org/10.3102/0034654308325583

Martin, D. B. (2008). E(race)ing race from a national conversation on mathematics teaching and learning: The National Mathematics Advisory Panel as White institutional space. The Mathematics Enthusiast, 5(2), 387-398.

https://scholarworks.umt.edu/tme/vol5/iss $2 / 20$ ?utm_source=scholarworks.umt.edu $\% 2 \mathrm{Ftme} \% 2 \mathrm{Fvol} 5 \% 2 \mathrm{Fiss} 2 \% 2 \mathrm{~F}$ 20\&utm medium=PDF\&utm campaign=PDFCoverPages

Martin, D. B. (2013). Race, racial projects, and mathematics education. Journal for Research in Mathematics Education, 44(1), 316-333. https://doi.org/10.5951/jresematheduc.44.1.0316

Martin, D. B. (2015). The collective Black and principles to actions. Journal of Urban Mathematics Education, 8(1), $17-$ 23.

Martin, D. B. (2019). Equity, inclusion, and antiBlackness in mathematics education. Race, Ethnicity, and Education, 22(4), 459-478. https://doi.org/10.1080/13613324.2019.1592833

Martin, D. B., Gholson, M. L., \& Leonard, J. (2010). Mathematics as gatekeeper: Power and privilege in the production of knowledge. Journal of Urban Mathematics Education, 3(2), 12-24. https://doi.org/10.21423/jume-v3i2a95

Maslow, A. H. (1943). Preface to motivation theory. Psychosomatic Medicine, 5, 85-92. https://doi.org/10.1097/00006842-194301000-00012

Matthews, J. S. (2018). When am I ever going to use this in the real world? Cognitive flexibility and urban adolescents' negotiation of the value of mathematics. Journal of Educational Psychology, 110(5), 726746. https://doi.org/10.1037/edu0000242

Miles, M. B., \& Huberman, A. M. (1994). Qualitative data analysis: An expanded sourcebook (2nd ed.). Sage Publications.

Mitchell, K. (2013). Race, difference, meritocracy, and English: Majoritarian stories in the education of secondary multilingual learners. Race Ethnicity and Education, 16(3), 339-364.

https://doi.org/10.1080/13613324.2011.645569 
Mo, Y., \& Singh, K. (2008). Parents' relationships and involvement: Effects on students' school engagement and performance. RMLE Online, 31(10), 1-11. https://doi.org/10.1080/19404476.2008.11462053

Morrison, G. M., Cosden, M. A., O'Farrell, S. L, \& Campos, E. (2003). Changes in Latino students' perceptions of school belonging over time: Impact of language proficiency, self-perceptions and teacher evaluations. The California School Psychologist, 8, 87-98. https://doi.org/10.1007/BF03340898

Morton, T. R., Gee, D. S., \& Woodson, A. N. (2020). Being vs. becoming: Transcending STEM identity development through Afropessimism, moving toward a Black X consciousness in STEM. The Journal of Negro Education, 88(3), 327-342. https://doi.org/10.7709/jnegroeducation.88.3.0327

Musu-Gillette, L., Robinson, J., McFarland, J., KewalRamani, A., Zhang, A., \& Wilkinson-Flicker, S. (2016). Status and trends in the education of racial and ethnic groups 2016. National Center for Education Statistics, U.S. Department of Education. https://nces.ed.gov/pubs2016/2016007.pdf

Nasir, N. S., \& Shah, N. (2011). On defense: African American males making sense of racialized narratives in mathematics education. Journal of African American Males in Education, 2(1), 24-45.

National Center for Education Statistics. (2020). Degrees conferred by race and sex. Institute of Educational Sciences. https://nces.ed.gov/fastfacts/display.asp?id=72

Oakes, J. (2005). Keeping track: How schools structure inequality ( $2^{\text {nd }}$ ed.). Yale University Press.

Okeke, N. A., Howard, L. C., Kurtz-Costes, B., \& Rowley, S. J. (2009). Academic race stereotypes, academic selfconcept, and racial centrality in African American youth. Journal of Black Psychology, 35(3), 366-387. https://doi.org/10.1177/0095798409333615

Osterman, K. F. (2000). Students' need for belonging in the school community. Review of Educational Research, 70(3), 323-367. https://doi.org/10.3102/00346543070003323

Paris, D. (2012). Culturally sustaining pedagogy: A needed change in stance, terminology, and practice. Educational Researcher, 41(3), 93-97. https://doi.org/10.3102/0013189X12441244

Patrick, H., Kaplan, A., \& Ryan, A. M. (2011). Positive classroom motivational environments: Convergence between mastery goal structure and classroom social climate. Journal of Educational Psychology, 103(2), 367382. https://doi.org/10.1037/a0023311

Pauker, K., Apfelbaum, E. P., \& Spitzer, B. (2015). When societal norms and social identity collide: The race talk dilemma for racial minority children. Social Psychological and Personality Science, 6(8), 887-895. https://doi.org/10.1177/1948550615598379

Pena-Shaff, J. B., Bessette-Symons, B., Tate, M., \& Fingerhut, J. (2019). Racial and ethnic differences in high school students' perceptions of school climate and disciplinary practices. Race Ethnicity and Education, 22(2), 269-284. https://doi.org/10.1080/13613324.2018.1468747

Pianta, R. C., Hamre, B. K., \& Mintz, S. (2012). Classroom Assessment Scoring System: Secondary manual. University of Virginia.

Remler, D. K., \& Van Ryzin, G. G. (2010). Research methods in practice: Strategies for description and causation. Sage Publications.

Reyes, A. H. (2006). Discipline, achievement, and race: Is zero tolerance the answer? Rowman and Littlefield.

Rouland, K. K., Matthews, J. S., Meyer, R. L., Byrd, C., \& Rowley, S. J. (2014). Culture clash? Interactions between Afro-cultural and mainstream cultural styles in classrooms serving African-American students. Interdisciplinary Journal of Teaching and Learning, 4(3), 186-202.

Ryan, A. M., \& Patrick, H. (2001). The classroom social environment and changes in adolescents' motivation and engagement during middle school. American Educational Research Journal, 38(2), 437-460. https://doi.org/10.3102/00028312038002437

Ryan, R. M. (1995). Psychological needs and the facilitation of integrative processes. Journal of Personality, 63(3), $397-$ 427. https://doi.org/10.1111/j.1467-6494.1995.tb00501.x

Saldaña, J. (2009). The coding manual for qualitative researchers. Sage Publications.

Sánchez, B., Colón, Y., \& Esparza, P. (2005). The role of school belonging and gender in the academic adjustment of Latino adolescents. Journal of Youth and Adolescence, 34(6), 619-628. https://doi.org/10.1007/s10964-005-89504 
Scott, J., Moses, M. S., Finnigan, K. S., Trujillo, T., \& Jackson, D. D. (2017). Law and order in school and society: How discipline and policing policies harm students of color, and what we can do about it. National Education Policy Center. https://nepc.colorado.edu/publication/law-and-order

Slaten, C., Ferguson, J., Allen, K., \& Vella-Brodrick, D. (2016). School belonging: A review of the history, current trends, and future directions. The Educational and Developmental Psychologist, 33(1), 1-15. https://doi.org/10.1017/edp.2016.6

Sleeter, C. E., \& Grant, C. A. (1991). Race, class, gender, and disability in current textbooks. In M. W. Apple \& L. K. Christian-Smith (Eds.), The politics of the textbook (pp. 78-110). Routledge.

Smerdon, B. A. (2002). Students' perceptions of membership in their high schools. Sociology of Education, 75(4), $287-$ 305. https://doi.org/10.2307/3090280

Solórzano, D. G., \& Ornelas, A. (2004). A critical race analysis of Latina/o and African American Advanced Placement enrollment in public high schools. The High School Journal, 87(3), 15-26. https://doi.org/10.1353/hsj.2004.0003

Stevens, T., Hamman, D., \& Olivarez, A. (2007). Hispanic students' perception of White teachers' mastery goal orientation influences sense of school belonging. Journal of Latinos and Education, 6(1), 55-70. https://doi.org/10.1080/15348430709336677

Stinson, D. W. (2004). Mathematics as "gate-keeper"(?): Three theoretical perspectives that aim toward empowering all children with a key to the gate. The Mathematics Educator, 14(1), 8-18.

https://scholarworks.gsu.edu/cgi/viewcontent.cgi?article=1018\&context=msit facpub

Stinson, D. W. (2013). Negotiating the "White male math myth": African American male students and success in school mathematics. Journal for Research in Mathematics Education, 44(1), 69-99. https://doi.org/10.5951/jresematheduc.44.1.0069

Strauss, A., \& Corbin, J. M. (1990). Basics of qualitative research: Grounded theory procedures and techniques. Sage Publications.

Strayhorn, T. L. (2020). Sense of belonging predicts persistence intentions among diverse dental education students: A multi-institutional investigation. Journal of Dental Education, 84(10), 1136-1142. https://doi.org/10.1002/jdd.12243

Umaña-Taylor, A. J., Quintana, S. M., Lee, R. M., Cross, W. E. Jr., Rivas-Drake, D., Schwartz, S. J., Syed, M., Yip, T., Seaton, E., \& Ethnic and Racial Identity in the 21st Century Study Group. (2014). Ethnic and racial identity during adolescence and into young adulthood: An integrated conceptualization. Child Development, 85(1), 21-39. https://doi.org/10.1111/cdev.12196

U.S. Census Bureau. (2010). State and county quick facts: Essex County, NJ: 2010 [Data set]. http://quickfacts.census.gov

Voelkl, K. E. (1996). Measuring students' identification with school. Educational and Psychological Measurement, 56(5), 760-770. https://doi.org/10.1177/0013164496056005003

Wallace, T. L., Ye, F., \& Chhuon, V. (2012). Subdimensions of adolescent belonging in high school. Applied Developmental Science, 16(3), 122-139. https://doi.org/10.1080/10888691.2012.695256

Walton, G. M., \& Brady, S. T. (2017). The many questions of belonging. In A. J. Elliot, C. S. Dweck, \& D. S. Yeager (Eds.), Handbook of competence and motivation: Theory and application (p. 272-293). The Guilford Press.

Walton, G. M., \& Cohen, G. L. (2007). A question of belonging: Race, social fit, and achievement. Journal of Personality and Social Psychology, 92(1), 82-96. https://doi.org/10.1037/0022-3514.92.1.82

Walton, G. M., \& Cohen, G. L. (2011). A brief social-belonging intervention improves academic and health outcomes of minority students. Science, 331(6023), 1447-1451. https://doi.org/10.1126/science.1198364

Wang, M.-T., \& Eccles, J. S. (2012). Social support matters: Longitudinal effects of social support on three dimensions of school engagement from middle to high school. Child Development, 83(3), 877-895.

https://doi.org/10.1111/j.1467-8624.2012.01745.x

Ware, F. (2006). Warm demander pedagogy: Culturally responsive teaching that supports a culture of achievement for African American students. Urban Education, 41(4), 427-456. https://doi.org/10.1177/0042085906289710

Wentzel, K. R. (1998). Social relationships and motivation in middle school: The role of parents, teachers, and peers. Journal of Educational Psychology, 90(2), 202-209. https://doi.org/10.1037/0022-0663.90.2.202

Wentzel, K. R. (1999). Social-motivational processes and interpersonal relationships: Implications for understanding motivation at school. Journal of Educational Psychology, 91(1), 76-97. https://doi.org/10.1037/0022$\underline{0663.91 .1 .76}$ 